\title{
SOLVING DIFFERENCE EQUATIONS IN SEQUENCES: UNIVERSALITY AND UNDECIDABILITY
}

\author{
GLEB POGUDIN $^{1}$, THOMAS SCANLON ${ }^{2}$ and MICHAEL WIBMER ${ }^{3}$ \\ ${ }^{1}$ Department of Computer Science, National Research University Higher School of Economics, \\ Moscow, Russia; \\ email: gleb.pogudin@lix.polytechnique.fr \\ ${ }^{2}$ University of California at Berkeley, Department of Mathematics, Berkeley, USA; \\ email: scanlon@math.berkeley.edu \\ ${ }^{3}$ Institute of Analysis and Number Theory, Graz University of Technology, Graz, Austria; \\ email: wibmer@math.tugraz.at
}

Received 12 September 2019; accepted 6 March 2020

\begin{abstract}
We study solutions of difference equations in the rings of sequences and, more generally, solutions of equations with a monoid action in the ring of sequences indexed by the monoid. This framework includes, for example, difference equations on grids (for example, standard difference schemes) and difference equations in functions on words. On the universality side, we prove a version of strong Nullstellensatz for such difference equations under the assumption that the cardinality of the ground field is greater than the cardinality of the monoid and construct an example showing that this assumption cannot be omitted. On the undecidability side, we show that the following problems are undecidable:

- testing radical difference ideal membership or, equivalently, determining whether a given difference polynomial vanishes on the solution set of a given system of difference polynomials;

- determining consistency of a system of difference equations in the ring of real-valued sequences;

- determining consistency of a system of equations with action of $\mathbb{Z}^{2}, \mathbb{N}^{2}$, or the free monoid with two generators in the corresponding ring of sequences over any field of characteristic zero.
\end{abstract}

2010 Mathematics Subject Classification: 12H10 (primary); 39A10, 13P25, 14Q20, 68Q40, 03D35 (secondary)

\section{Introduction}

An ordinary difference ring $(A, \sigma)$ is a commutative ring $A$ equipped with a distinguished ring endomorphism $\sigma: A \rightarrow A$. The most basic example of a

(C) The Author(s) 2020. This is an Open Access article, distributed under the terms of the Creative Commons Attribution licence (http://creativecommons.org/licenses/by/4.0/), which permits unrestricted re-use, distribution, and reproduction in any medium, provided the original work is properly cited. 
difference ring is the ring $\mathbb{C}^{\mathbb{N}}$ of sequences of complex numbers with $\sigma$ defined by $\left(a_{i}\right)_{i \in \mathbb{N}} \mapsto\left(a_{i+1}\right)_{i \in \mathbb{N}}$. More generally, if $\phi: X \rightarrow X$ is any self-map on a set $X$ and $A$ is the ring of complex-valued functions on $X$, then $\sigma: A \rightarrow A$ defined by $f \mapsto f \circ \phi$ is a difference ring. The special case where $X=\mathbb{R}$ is the real line and $\phi$ is given by $\phi(x)=x+1$ gives the operator defined by $f(t) \mapsto f(t+1)$ and explains the origin of the name 'difference ring' in that the discrete difference operator $\Delta$ defined by $f(t) \mapsto f(t+1)-f(t)$ may be expressed as $\Delta=\sigma-$ id. Generalizing to allow for additional operators, we might consider partial difference rings $\left(A, \sigma_{1}, \ldots, \sigma_{n}\right)$ with several distinguished ring endomorphisms $\sigma_{j}: A \rightarrow A$. Natural instances of such partial difference rings with commuting operators include rings of sequences indexed by $n$-tuples of natural numbers and the rings of $n$-variable functions. There are also natural examples of such partial difference rings with noncommuting difference operators coming from number theory, the theory of iterated function systems, and symbolic dynamics.

We may think of a partial difference ring $\left(A, \sigma_{1}, \ldots, \sigma_{n}\right)$ as the $\operatorname{ring} A$ given together with an action by ring endomorphisms of $M_{n}$, the free monoid on $n$ generators. If we require that these operators commute, then this may be seen as an action by $\mathbb{N}^{n}$. Likewise, if we require that the operators are, in fact, ring automorphisms, then it is an action by $F_{n}$, the free group on $n$-generators.

As with algebraic and differential equations, the most basic problems for difference equations come down to solving these equations in some specified difference ring. As a preliminary, difficult subproblem, one must determine whether the equations under consideration admit any solutions at all. In the optimal cases, solvability of a system of equations is equivalent to a suitable Nullstellensatz in some associated ring of polynomials (respectively, differential polynomials or difference polynomials). While in the case of polynomial equations in finitely many variables, these problems admit well-known solutions, for difference and differential equations and their relatives, there are subtle distinctions between those problems that may be solved and those for which no algorithm exists.

In many cases, the problems we are considering may be resolved by analyzing the associated first-order theories. The prototypical decidability theorems for equations are Tarski's theorems on the decidability and completeness of the theories of real closed fields and of algebraically closed fields of a fixed characteristic [28]. This logical theorem is complemented algebraically by Hilbert's Nullstellensatz, which gives a precise sense in which implications for systems of polynomial equations may be expressed in terms of ideal membership problems. 
Theorems analogous to Tarski's are known for difference and differential fields. The theories of difference fields, of differential fields of characteristic zero, and even of partial differential fields of characteristic zero and of differencedifferential fields of characteristic zero are known to have model companions (see [3-5, 19]). Moreover, for each of these theories, quantifier simplification theorems (and even full quantifier elimination theorems in the case of differential fields) are known. From these results, one may deduce on general grounds the existence of algorithms for determining the consistency of systems of difference (respectively, differential or difference-differential) equations in such fields and explicitly, if not always efficient, such algorithms may be extracted from the more geometric presentations of the axioms. Better algorithms based on characteristic set methods are known [8, 9, 17].

From the algebraic point of view, the consistency checking problem may be expressed in terms of some form of a Nullstellensatz. For example, the weak form of the classical Nullstellensatz of Hilbert says that if $K$ is an algebraically closed field and $f_{1}, \ldots, f_{\ell} \in K\left[x_{1}, \ldots, x_{n}\right]$ is a sequence of polynomials in the finitely many variables $x_{1}, \ldots, x_{n}$, then the system of equations

$$
f_{1}(\mathbf{x})=\cdots=f_{\ell}(\mathbf{x})=0
$$

(where we have written $\left.\mathbf{x}=\left(x_{1}, \ldots, x_{n}\right)\right)$ has a solution in $K$ if and only if 1 does not belong to the ideal $\left\langle f_{1}, \ldots, f_{\ell}\right\rangle$ generated by $f_{1}, \ldots, f_{\ell}$. The latter condition can be verified by a linear algebra computation (see [14] and references therein).

Hilbert's Nullstellensatz takes a stronger form in that one may reduce implications between systems of equations to explicit computations in polynomial rings. That is, given equations as above and $g \in K[\mathbf{x}]$ being any polynomial, then $g$ vanishes on every solution to Equation (1) if and only if $g \in \sqrt{\left\langle f_{1}, \ldots, f_{\ell}\right\rangle}$, the radical of the ideal generated by $f_{1}, \ldots, f_{\ell}$. Similar results are known for equations in differential, difference, and difference-differential fields. The situation is murkier if we consider partial difference equations, that is, difference equations with respect to several distinguished ring endomorphisms. It is noted in [12] that the theory of difference fields with respect to finitely many distinguished endomorphisms has a model companion, and, in fact, a simple variant of the method for determining the consistency of systems of difference equations for ordinary difference equations extends to this case of partial difference equations. However, if the distinguished endomorphisms are required to commute, then no such model companion exists [15].

Rings of sequences are among the most natural places to look for solutions of difference equations. In particular, algorithms for detecting the solvability of finite systems of difference equations in sequence rings are available [24]. However, the general problem of solving equations in sequences is much more complicated than 
the analogous problem for difference fields: whenever $K$ is infinite, the first-order theory of the sequence ring $K^{\mathbb{N}}$ regarded in the language of difference rings is undecidable [13, Proposition 3.5].

The staring point for us was a recent paper [24] that contains the following results about solving difference equations in sequences:

- The weak Nullstellensatz [24, Theorem 7.1]: for any algebraically closed difference field $(K, \sigma)$ and a finite set $S$ of difference equations over $K$, there is a solution in $K^{\mathbb{N}}$ to the system $S$ if and only if the difference ideal generated by $S$ is proper.

- An effective bound [24, Theorem 3.4] that yields an algorithm for deciding whether a difference ideal given by its generators is proper and, consequently, an algorithm for deciding consistency of a finite system of difference equations in $K^{\mathbb{N}}$.

Remarkably, while the proof of the weak difference Nullstellensatz is rather routine for $K$ uncountable, the result holds for arbitrary $K$.

In this paper, we answer several natural questions aimed at extending the above results about solving difference equations in sequences.

QUESTION 1 (weak Nullstellensatz $\rightarrow$ strong Nullstellensatz). If $f_{1}, \ldots, f_{\ell}$, and $g$ are difference polynomials over an algebraically closed difference field $K$ and $g$ vanishes on every solution to the system of difference equations $f_{1}(\mathbf{x})=\cdots=$ $f_{\ell}(\mathbf{x})=0$ in $K^{\mathbb{N}}$, must $g$ belong to the radical of the difference ideal generated by $f_{1}, \ldots, f_{\ell}$ ?

ANSWER. Depends on the cardinality of $K$ (Theorems 3.1 and 3.2).

More precisely, we show that the answer is Yes if $K$ is uncountable (Theorem 3.1) and give an example that shows that the answer is No for $K=\overline{\mathbb{Q}}$ (Theorem 3.2). It is interesting to compare this result with the weak Nullstellensatz [24, Theorem 7.1] that holds for a ground field of any cardinality, but the proof for the countable case is much harder than the proof for the uncountable case.

QUESTION 2 (testing consistency $\rightarrow$ testing radical difference ideal membership). Is there an algorithm that, given difference polynomials $f_{1}, \ldots, f_{\ell}$, and $g$, decides whether $g$ belongs to the radical difference ideal generated by $f_{1}, \ldots, f_{\ell}$ ?

ANSWER. No (Theorem 3.7).

This result contrasts not only with the existence of an algorithm for this problem if $g=1$ (see [24, Theorem 3.4]) but also with the decidability of the membership 
problem for radical differential ideals [25, page 110]. Furthermore, we are aware of only one prior undecidability result for the membership problem in the context of differential/difference algebra [29], and this result holds if one considers not necessarily radical ideals and at least two derivations.

QUESTION 3 (not necessarily algebraically closed $K$ ). Is there an algorithm that, given difference polynomials $f_{1}, \ldots, f_{\ell}$ over $\mathbb{R}$, decides whether the system $f_{1}=\cdots=f_{\ell}=0$ has a solution in $\mathbb{R}^{\mathbb{N}}$ ?

ANSWER. No (Theorem 3.6).

Moreover, Theorem 3.6 shows that the answer is No if we replace $\mathbb{R}$ with any subfield of $\mathbb{R}$ (including $\mathbb{Q}$ ). Again, the situation is different compared to the differential case: The problem of deciding the existence of a real analytic solution of a system of differential equations over $\mathbb{Q}$ is decidable [27, Section 4].

QUESTION 4 (index monoids other than $\mathbb{N}$ or $\mathbb{Z}$ ). Is there an algorithm for deciding consistency of systems of difference equations with respect to actions of $\mathbb{N}^{2}$ or the free monoid with two generators when the solutions are sought in the sequences indexed by the corresponding monoid?

ANSWER. No (Propositions 3.9 and 3.10).

Notably, the problem of the solvability of equations in the free monoid itself is decidable [21].

One of the crucial technical ingredients (used to prove Theorems 3.2 and 3.7 and Proposition 3.10) is Lemma 4.6, which connects the membership problem for a radical difference ideal to a problem of Skolem-Mahler-Lech [7, Section 2.3] type for piecewise polynomial maps. For related undecidability results for dynamical systems associated with other types of maps, see [2, 16, 23] and references therein.

\section{Preliminaries}

Throughout the paper, $\mathbb{N}$ denotes the set of nonnegative integers.

2.1. Difference rings and equations. The main objects of the paper are difference equations and their generalizations. A detailed introduction to difference rings can be found in $[6,20]$. 
DEFINITION 2.1 (Difference rings). A difference ring is a pair $(A, \sigma)$, where $A$ is a commutative ring and $\sigma: A \rightarrow A$ is a ring endomorphism. We often abuse notation saying that $A$ is a difference ring when we mean the pair $(A, \sigma)$.

The following example of a difference ring will be central in this paper.

EXAMPLE 2.2 (Ring of sequences). If $R$ is any commutative ring, then the sequence rings $R^{\mathbb{N}}$ and $R^{\mathbb{Z}}$ (with componentwise addition and multiplication) are difference rings with $\sigma$ defined by $\sigma\left(\left(x_{i}\right)_{i \in \mathbb{N}}\right):=\left(x_{i+1}\right)_{i \in \mathbb{N}}\left(\sigma\left(\left(x_{i}\right)_{i \in \mathbb{Z}}\right):=\right.$ $\left(x_{i+1}\right)_{i \in \mathbb{Z}}$, respectively).

DEFINITION 2.3 (Difference polynomials). Let $A$ be a difference ring.

- The free difference $A$-algebra in one generator $X$ over $A$, also called the ring of difference polynomials in $X$ over $A$, may be realized as the ordinary polynomial ring, $A\left[\sigma^{j}(X) \mid j \in \mathbb{N}\right]$, in the indeterminates $\left\{\sigma^{j}(X) \mid j \in \mathbb{N}\right\}$ with the action $\sigma\left(\sigma^{j}(X)\right):=\sigma^{j+1}(X)$.

- Similarly, for $\boldsymbol{X}=\left(X_{1}, \ldots, X_{n}\right)$, one obtains the difference polynomial ring $A\left[\sigma^{j}(\boldsymbol{X}) \mid j \in \mathbb{N}\right]$ in $n$ variables.

DEFinition 2.4. If $(A, \sigma)$ is a difference ring and $F \subseteq A\left[\sigma^{j}(\boldsymbol{X}) \mid j \in \mathbb{N}\right]$, where $\boldsymbol{X}=\left(X_{1}, \ldots, X_{n}\right)$ is a set of difference polynomials over $A,(A, \sigma) \rightarrow(B, \sigma)$ is a map of difference rings, and $\mathbf{x}=\left(x_{1}, \ldots, x_{n}\right) \in B^{n}$ is an $n$-tuple from $B$, then we say that $\mathbf{x}$ is a solution of the system $F=0$ if, under the unique map of difference rings $A\left[\sigma^{j}(\boldsymbol{X}) \mid j \in \mathbb{N}\right] \rightarrow B$ given by extending the given map $A \rightarrow B$ and sending $X_{i} \mapsto x_{i}$ for $1 \leqslant i \leqslant n$, every element of $F$ is sent to 0 .

EXAMPLE 2.5 (Fibonacci numbers). Consider the Fibonacci sequence $f:=$ $(1,1,2,3,5, \ldots) \in \mathbb{C}^{\mathbb{N}}$. Then the fact that the sequence satisfies a recurrence $f_{n+2}=f_{n+1}+f_{n}$ can be expressed by saying that $\boldsymbol{f}$ is a solution of a difference equation $\sigma^{2}(X)-\sigma(X)-X=0$, where $\sigma^{2}(X)-\sigma(X)-X \in \mathbb{C}\left[\sigma^{j}(X) \mid j \in \mathbb{N}\right]$.

2.2. Rings with a monoid action and equations. In this paper, we will often be interested in rings of 'sequences' that would generalize Example 2.2 to sequences indexed by $\mathbb{Z}^{2}$ (for example, difference schemes for partial differential equations) or any other semigroup.

Definition 2.6 ( $M$-rings). Let $M$ be a monoid. A pair $(A, \sigma)$ where $A$ is a commutative ring and $\sigma$ is an action of $M$ on $A$ by endomorphisms is called 
an $M$-ring. For every $a \in A$ and $m \in M$, we define the image of $a$ under the endomorphism corresponding to $m$ by $\sigma^{m}(a)$.

We note that every difference ring is an $\mathbb{N}$-ring for the monoid $(\mathbb{N},+)$. A morphism of $M$-rings is a morphism of rings that commutes with the $M$-action.

EXAMPLE 2.7 (Rings of sequences indexed by $\mathbb{N}^{2}$ and $\mathbb{Z}^{2}$ ). If $R$ is any commutative ring, then the rings $R^{\mathbb{N}^{2}}$ and $R^{\mathbb{Z}^{2}}$ are $\mathbb{N}^{2}$-rings with $\sigma$ defined by

$$
\sigma^{(1,0)}\left(\left(x_{i, j}\right)_{i, j \in \mathbb{N}}\right):=\left(x_{i+1, j}\right)_{i, j \in \mathbb{N}} \quad \text { and } \quad \sigma^{(0,1)}\left(\left(x_{i, j}\right)_{i, j \in \mathbb{N}}\right):=\left(x_{i, j+1}\right)_{i, j \in \mathbb{N}} .
$$

The action on $R^{\mathbb{Z}^{2}}$ is defined analogously.

EXAMPLE 2.8. In general, if $R$ is a commutative ring and $M$ a monoid, then the ring $R^{M}$ of $M$-sequences is the commutative ring of all maps from $M$ to $R$ (with componentwise addition and multiplication) and action given by

$$
\sigma^{m}\left(\left(x_{\ell}\right)_{\ell \in M}\right)=\left(x_{\ell m}\right)_{\ell \in M}
$$

for $m \in M$.

The following example is a special case of Example 2.8.

EXAMPLE 2.9 (Functions on words). Let $\Sigma$ be a finite alphabet. By $\left(\Sigma^{*}, \cdot\right)$ we denote the monoid of all words in $\Sigma$ with the operation of concatenation. Let $R$ be a commutative ring. Consider the ring of functions $R^{\Sigma^{*}}$ from $\Sigma^{*}$ to $R$ that we will identify with the ring of $\Sigma^{*}$-indexed sequences. Then $R^{\Sigma^{*}}$ can be endowed with a structure of $\Sigma^{*}$ ring as follows

$$
\sigma^{w}\left(\left(x_{u}\right)_{u \in \Sigma^{*}}\right):=\left(x_{u w}\right)_{u \in \Sigma^{*}} \text { for every } w \in \Sigma^{*} .
$$

Definition 2.10 ( $M$-polynomials). We fix a monoid $M$. Let $A$ be an $M$-ring.

- The free $M$-algebra over $A$ in one generator $X$ over $A$, also called the ring of $M$-polynomials in $X$ over $A$, may be realized as the ordinary polynomial ring, $A\left[\sigma^{m}(X) \mid m \in M\right]$, in the indeterminates $\left\{\sigma^{m}(X) \mid m \in M\right\}$ with the action $\sigma^{m_{1}}\left(\sigma^{m_{2}}(X)\right):=\sigma^{m_{1} m_{2}}(X)$ for every $m_{1}, m_{2} \in M$.

- Similarly, for $\boldsymbol{X}=\left(X_{1}, \ldots, X_{n}\right)$, one obtains the ring of $M$-polynomials $A\left[\sigma^{m}(\boldsymbol{X}) \mid m \in M\right]$ in $n$ variables. 
DEFINITION 2.11. We fix a monoid $M$. If $(A, \sigma)$ is an $M$-ring and $F \subseteq$ $A\left[\sigma^{m}(\boldsymbol{X}) \mid m \in M\right]$, where $\boldsymbol{X}=\left(X_{1}, \ldots, X_{n}\right)$ is a set of $M$-polynomials over $A,(A, \sigma) \rightarrow(B, \sigma)$ is a map of $M$-rings, and $\mathbf{x}=\left(x_{1}, \ldots, x_{n}\right) \in B^{n}$ is an $n$-tuple from $B$, then we say that $\mathbf{x}$ is a solution of the system $F=0$ if, under the unique map of $M$-rings $A\left[\sigma^{m}(\boldsymbol{X}) \mid m \in M\right] \rightarrow B$ given by extending the given map $A \rightarrow B$ and sending $X_{i} \mapsto x_{i}$ for $1 \leqslant i \leqslant n$, every element of $F$ is sent to 0 . For $f \in A\left[\sigma^{m}(\boldsymbol{X}) \mid m \in M\right]$, we denote the image of $f$ under the above map by $f(\mathbf{x})$.

EXAMPLE 2.12 (Discrete harmonic functions). Consider a $\mathbb{C}$-valued function $\boldsymbol{x}=\left(x_{i, j}\right)_{i, j \in \mathbb{Z}^{2}}$ on the integer lattice. It is called a discrete harmonic function [11] if, for every $i, j \in \mathbb{Z}^{2}, 4 x_{i, j}=x_{i+1, j}+x_{i-1, j}+x_{i, j+1}+x_{i, j-1}$. The fact that it is a discrete harmonic function can be expressed by the fact that it is a solution of the following $\mathbb{Z}^{2}$-polynomial

$$
4 X-\sigma^{(1,0)}(X)-\sigma^{(-1,0)}(X)-\sigma^{(0,1)}(X)-\sigma^{(0,-1)}(X) \in \mathbb{C}\left[\sigma^{m}(X) \mid m \in \mathbb{Z}^{2}\right] .
$$

EXAMPLE 2.13. Let $M=\{a, b\}^{*}$ be a monoid of binary words with respect to concatenation. Then the fact that a function $d: M \rightarrow \mathbb{R}$ is a martingale [26, page 2] can be expressed by the fact that $d$ is a solution of the following $M$ polynomial

$$
X-\frac{1}{2} \sigma^{a}(X)-\frac{1}{2} \sigma^{b}(X) \in \mathbb{C}\left[\sigma^{m}(X) \mid n \in M\right] .
$$

\section{Main results}

3.1. Universality of sequence rings. Let $M$ be a monoid, let $k$ be a field, and let $\boldsymbol{X}=\left(X_{1}, \ldots, X_{n}\right)$. For a subset $F$ of $k\left[\sigma^{m}(X) \mid m \in M\right]$, we let

$$
\mathcal{V}(F)=\left\{\mathbf{x} \in\left(k^{M}\right)^{n} \mid f(\mathbf{x})=0 \forall f \in F\right\}
$$

denote the set of solutions of $F$ in $k^{M}$ and for a subset $S$ of $\left(k^{M}\right)^{n}$, we let

$$
\mathcal{I}(S)=\left\{f \in k\left[\sigma^{m}(\boldsymbol{X}) \mid m \in M\right] \mid f(\boldsymbol{x})=0 \forall \boldsymbol{x} \in S\right\}
$$

denote the set of all $M$-polynomials vanishing on $S$.

THEOREM 3.1 (Strong Nullstellensatz). Let $M$ be a monoid, let $k$ be an algebraically closed field such that $|k|>|M|$, and let $\boldsymbol{X}=\left(X_{1}, \ldots, X_{n}\right)$. Then, for every subset $F$ of $k\left[\sigma^{m}(X) \mid m \in M\right]$, we have

$$
\mathcal{I}(\mathcal{V}(F))=\sqrt{\left\langle\sigma^{m}(F) \mid m \in M\right\rangle} .
$$

The following theorem shows that the condition $|k|>|M|$ in Theorem 3.1 cannot be omitted. 
THEOREM 3.2. There exists a finite set $F$ of difference equations over $\overline{\mathbb{Q}}$ such that

$$
\mathcal{I}(\mathcal{V}(F)) \supsetneqq \sqrt{\left\langle\sigma^{i}(F) \mid i \in \mathbb{N}\right\rangle} \text {. }
$$

REMARK 3.3 (Weak Nullstellensatz). Theorems 3.1 and 3.2 complement the weak Nullstellensatz from [24] in a surprising way. Theorem 7.1 in [24] established the weak Nullstellensatz for $M=\mathbb{N}$, that is,

$$
\mathcal{I}(\mathcal{V}(F))=\varnothing \Longleftrightarrow 1 \in \sqrt{\left\langle\sigma^{m}(F) \mid m \in M\right\rangle}
$$

without any restrictions on the cardinality of $k$. However, the proof for the case of uncountable $k$ (see [24, Proposition 6.3]) was much simpler than the proof of the general statement. Our results indicate that this difference between the countable and uncountable cases is not an artifact of the proof in [24] but rather a conceptual distinction.

COROLLARY 3.4 (Universality of the ring of sequences). Let $M$ be a monoid, let $k$ be an algebraically closed field such that $|k|>|M|$, and let $\boldsymbol{X}=\left(X_{1}, \ldots, X_{n}\right)$. Then, for every subset $F$ of $k\left[\sigma^{m}(\boldsymbol{X}) \mid m \in M\right]$ and $g \in k\left[\sigma^{m}(\boldsymbol{X}) \mid m \in M\right]$, the following are equivalent:

- $g=0$ holds for every solution of $F=0$ in any reduced $M$-ring containing $k$;

- $g=0$ holds for every solution of $F=0$ in $k^{M}$.

Proof. If the latter point holds, then $g^{e} \in\left\langle\sigma^{m}(F) \mid m \in M\right\rangle$ for some $e \geqslant 1$ by Theorem 3.1. Thus for every solution $\boldsymbol{x}$ in some reduced $M$-ring containing $k$, we have $g(\boldsymbol{x})^{e}=0$ and therefore $g(\boldsymbol{x})=0$ as desired.

REMARK 3.5 (Nonconstant $k$ ). Moreover, we prove a more general theorem (Theorem 4.1) than Theorem 3.1, where the field $k$ is not necessarily constant. We also establish an alternative formulation of the strong difference Nullstellensatz that works without any assumptions on the base difference field $k$ (Theorem 4.2).

\subsection{Undecidability results.}

THEOREM 3.6. For every field $k$ such that $k \subseteq \mathbb{R}$ and every computable subfield $k_{0} \subset k$, the following problem is undecidable: Given a finite system of difference equations with coefficients in $k_{0}$, determine whether it has a solution in $k^{\mathbb{N}}$ (respectively, $k^{\mathbb{Z}}$ ). 
THEOREM 3.7. Let $M$ be $\mathbb{N}$ or $\mathbb{Z}$, let $k$ be a field of characteristic zero, and let $k_{0} \subset k$ be a computable subfield. Then the following problem is undecidable: Given a finite system of difference equations $F=0$ and a difference equation $g=0$ with coefficients in $k_{0}$, determine whether $g=0$ holds for every solution on $F=0$ in $k^{M}$.

COROLlary 3.8. Let $M$ be $\mathbb{N}$ or $\mathbb{Z}$, let $k$ be a field of characteristic zero, and let $k_{0} \subset k$ be a computable subfield. Then the following problems are undecidable:

(P1) Given $f_{1}, \ldots, f_{\ell}, g \in k_{0}\left[\sigma^{m}(\boldsymbol{X}) \mid m \in M\right]$, where $\boldsymbol{X}=\left(X_{1}, \ldots, X_{n}\right)$, determine whether the system $f_{1}=\cdots=f_{\ell}=0, g \neq 0$ has a solution in $k^{M}$.

(P2) Given $f_{1}, \ldots, f_{\ell}, g \in k_{0}\left[\sigma^{m}(\boldsymbol{X}) \mid m \in M\right]$, where $\boldsymbol{X}=\left(X_{1}, \ldots, X_{n}\right)$, determine whether

$$
g \in \sqrt{\left\langle\sigma^{m}\left(f_{1}\right), \ldots, \sigma^{m}\left(f_{\ell}\right) \mid m \in M\right\rangle} .
$$

PROPOSITION 3.9. Let $k$ be a field of characteristic zero and $k_{0} \subset k$ be a computable subfield, and let the monoid $M$ be either $\mathbb{N}^{2}$ or $\mathbb{Z}^{2}$. Then the following problem is undecidable: Given a finite set $F$ of $M$-polynomials over $k_{0}$, decide whether the system $F=0$ has a solution in $k^{M}$.

Proposition 3.10. Let $k$ be a field of characteristic zero and $k_{0} \subset k$ be a computable subfield, and let $M_{2}$ be a free monoid with two generators. Then the following problem is undecidable: Given a finite set $F$ of $M_{2}$-polynomials over $k_{0}$, decide whether $F=0$ has a solution in $k^{M_{2}}$.

\section{Proofs}

Throughout this section, we will use the following notation. For a tuple of sequences $\left(\left\{x_{1, i}\right\}_{i \in M}, \ldots,\left\{x_{n, i}\right\}_{i \in M}\right)$, we will denote $\boldsymbol{x}_{i}=\left(x_{1, i}, \ldots, x_{n, i}\right)$ for every $i \in M$, and the original tuple of sequences will be denoted by $\left\{\boldsymbol{x}_{i}\right\}_{i \in M}$.

4.1. Proof of Theorem 3.1. In this section, we establish two closely related versions of a strong difference Nullstellensatz (Theorems 4.1 and 4.2). Theorem 4.1 contains Theorem 3.1 as a special case.

We begin by introducing the notation necessary to state our general result. Let $M$ be a monoid and let $k$ be an $M$-field. We note that for any field extension $K$ of $k$, the map $k \rightarrow K^{M}, a \mapsto\left(\sigma^{m}(a)\right)_{m \in M}$ is a morphism of $M$-rings. Let 
$\mathbf{X}=\left(X_{1}, \ldots, X_{n}\right)$. As in Section 3.1, for a subset $F$ of $k\left[\sigma^{m}(\mathbf{X}) \mid m \in M\right]$, we set

$$
\mathcal{V}(F)=\left\{\boldsymbol{x} \in\left(k^{M}\right)^{n} \mid f(\boldsymbol{x})=0 \forall f \in F\right\},
$$

and for a subset $S$ of $\left(k^{M}\right)^{n}$, we set

$$
\mathcal{I}(S)=\left\{f \in k\left[\sigma^{m}(\mathbf{X}) \mid m \in M\right] \mid f(\boldsymbol{x})=0 \forall \boldsymbol{x} \in S\right\} .
$$

THEOREM 4.1 (Strong Nullstellensatz). Let $k$ be an algebraically closed $M$-field such that $|k|>|M|$. Then, for every subset $F$ of $k\left[\sigma^{m}(\mathbf{X}) \mid m \in M\right]$, we have

$$
\mathcal{I}(\mathcal{V}(F))=\sqrt{\left\langle\sigma^{m}(F) \mid m \in M\right\rangle} .
$$

In Section 4.6, we present an example that shows that the assumption $|k|>$ $|M|$ in Theorem 4.1 cannot be omitted. However, we also have an alternative formulation of Theorem 4.1 that works without any assumptions on the base difference field $k$. For a subset $F$ of $k\left[\sigma^{m}(\mathbf{X}) \mid m \in M\right]$, we set

$$
\begin{gathered}
\Im(F)=\left\{f \in k\left[\sigma^{m}(\mathbf{X}) \mid m \in M\right] \mid \text { for every field extension } K / k,\right. \\
\left.f \text { vanishes on all solutions of } F \text { in } K^{M}\right\} .
\end{gathered}
$$

THEOREM 4.2. Let $k$ be an $M$-field and $F \subseteq k\left[\sigma^{m}(\mathbf{X}) \mid m \in M\right]$. Then

$$
\Im(F)=\sqrt{\left\langle\sigma^{m}(F) \mid m \in M\right\rangle} .
$$

For the proofs of Theorems 4.1 and 4.2, we will need the following version of the strong algebraic Nullstellensatz for polynomials in infinitely many variables. Let $k$ be a field and $\mathbf{Y}$ a (not necessarily finite) set of indeterminates over $k$. For $F \subseteq k[\mathbf{Y}]$, we set

$$
\mathbb{V}(F)=\left\{\mathbf{y} \in k^{\mathbf{Y}} \mid f(\mathbf{y})=0 \forall f \in F\right\},
$$

and for $S \subseteq k^{\mathbf{Y}}$, we set

$$
\mathbb{I}(S)=\{f \in k[\mathbf{Y}] \mid f(\mathbf{y})=0 \forall \mathbf{y} \in S\} .
$$

LEMMA 4.3. Let $k$ be an algebraically closed field and $F \subseteq k[\mathbf{Y}]$. If $|k|>|\mathbf{Y}|$, then $\mathbb{I}(\mathbb{V}(F))=\sqrt{\langle F\rangle}$.

Proof. This follows from the main theorem of [18].

Proof of Theorem 4.1. As $\mathcal{I}(S)$ is a radical $M$-invariant ideal, for any subset $S$ of $k^{M}$, we have

$$
\sqrt{\left\langle\sigma^{m}(F) \mid m \in M\right\rangle} \subseteq \mathcal{I}(\mathcal{V}(F))
$$


To establish the reverse inclusion, we set $\mathbf{Y}=\left\{\sigma^{m}(\mathbf{X}) \mid m \in M\right\}$ so that $\left(k^{M}\right)^{n}$ can be identified with $k^{\mathbf{Y}}$. The nature of the map $k \rightarrow k^{M}, a \mapsto\left(\sigma^{m}(a)\right)_{m \in M}$ is such that for $f \in k\left[\sigma^{m}(\mathbf{X}) \mid m \in M\right]$ and $\mathbf{x} \in\left(k^{M}\right)^{n}$, we have $f(\boldsymbol{x})=0 \in\left(k^{M}\right)^{n}$ if and only if $\sigma^{m}(f)(\boldsymbol{x})=0 \in k$ for all $m \in M$. So, under the identification $\left(k^{M}\right)^{n}=k^{\boldsymbol{Y}}$, we have $\mathcal{V}(I)=\mathbb{V}(I)$ for any $M$-invariant ideal $I$ of

$$
k\left[\sigma^{m}(\mathbf{X}) \mid m \in M\right]=k[\boldsymbol{Y}] .
$$

Similarly, for any subset $S$ of $\left(k^{M}\right)^{n}=k^{\mathbf{Y}}$, we have

$$
f \in \mathcal{I}(S) \subseteq k\left[\sigma^{m}(\mathbf{X}) \mid m \in M\right]
$$

if and only if $\sigma^{m}(f) \in \mathbb{I}(S) \subseteq k[\mathbf{Y}]$ for all $m \in M$, in particular, $\mathcal{I}(S) \subseteq \mathbb{I}(S)$. Clearly $\mathcal{V}(F)=\mathcal{V}(I)$, where $I=\left\langle\sigma^{m}(F) \mid m \in M\right\rangle$, and so

$$
\mathcal{I}(\mathcal{V}(F))=\mathcal{I}(\mathcal{V}(I))=\mathcal{I}(\mathbb{V}(I)) \subseteq \mathbb{I}(\mathbb{V}(I))=\sqrt{I}
$$

In the case where $M$ is infinite, the last equality here follows from Lemma 4.3 since $|X|=n|M|=|M|<|k|$. In the case where $M$ is finite, the last equality reduces to the usual algebraic strong Nullstellensatz.

Proof of Theorem 4.2. Again, the inclusion $\sqrt{I} \subseteq \Im(F)$, where

$$
I=\left\langle\sigma^{m}(F) \mid m \in M\right\rangle,
$$

is clear. To establish the reverse inclusion, we let $K$ denote an algebraically closed field extension of $k$ with $|K|>|M|$ and we proceed similarly to the proof of Theorem 4.1: For $\boldsymbol{Y}=\left\{\sigma^{m}(\mathbf{X}) \mid m \in M\right\}$ we have, under the identification $\left(K^{M}\right)^{n}=K^{Y}$, that

$$
\begin{aligned}
\left\{\boldsymbol{x} \in\left(K^{M}\right)^{n} \mid f(\boldsymbol{x})\right. & =0 \forall f \in F\} \\
& =\left\{\boldsymbol{x} \in K^{\boldsymbol{Y}} \mid \sigma^{m}(f)(\boldsymbol{x})=0 \forall f \in F, m \in M\right\} .
\end{aligned}
$$

Thus, if $f \in \mathfrak{I}(F) \subset k\left[\sigma^{m}(\mathbf{X}) \mid m \in M\right]=k[\boldsymbol{Y}]$, then $f \in \mathbb{I}(\mathbb{V}(I))$. Note that here $I \subseteq k\left[\sigma^{m}(\mathbf{X}) \mid m \in M\right] \subseteq K[\boldsymbol{Y}]$, but $\mathbb{I}$ and $\mathbb{V}$ are applied with respect to $K$. So it follows from Lemma 4.3 that $f \in \sqrt{\langle I\rangle}$, where $\langle I\rangle \subseteq K[X]$. But $K[X]=$ $k[X] \otimes_{k} K$ and $\langle I\rangle=I \otimes_{k} K$. Therefore, if $e \geqslant 1$ is such that $f^{e} \in\langle I\rangle=I \otimes_{k} K$, then $f^{e} \in\left(I \otimes_{k} K\right) \cap k[X]=I$. Thus $f \in \sqrt{I}$ as desired.

4.2. Proof of Theorem 3.6. Let $M$ be $\mathbb{N}$ or $\mathbb{Z}$. For every polynomial equation $P\left(t_{1}, \ldots, t_{n}\right)=0$ with coefficients in $\mathbb{Z}$, we will construct a system of difference equations $F_{P}=0$ over $\mathbb{Q}$ such that $P=0$ has a solution in $\mathbb{Z}^{n}$ if and only if 
$F_{P}=0$ has a solution in $k^{M}$. Then the theorem will follow from the undecidability of Diophantine equations [22].

LEMMA 4.4. Let $\boldsymbol{Y}=\left(Y_{1}, \ldots, Y_{6}\right)$. There exists a finite set

$$
G \subset \mathbb{Q}\left[\sigma^{i}(X), \sigma^{i}(\boldsymbol{Y}) \mid i \in M\right]
$$

such that, for every solution of $G=0$ in $k^{M}$, the sequence $\left(x_{i}\right)_{i \in M}$ corresponding to $X$ has the property that $\left(x_{i}\right)_{i \in \mathbb{N}}$ contains infinitely many zeroes.

Moreover, for every sequence $\left(x_{i}\right)_{i \in M} \in k^{M}$ such that $\left(x_{i}\right)_{i \in \mathbb{N}}$ contains infinitely many zeroes, there exists a solution of $G=0$ in $k^{M}$ such that $\left(x_{i}\right)_{i \in M}$ is the $X$ coordinate of the solution.

Proof. We define $G$ as

$$
G:=\left\{X Y_{1}, Y_{2}-Y_{3}^{2}-Y_{4}^{2}-Y_{5}^{2}-Y_{6}^{2}, \sigma\left(Y_{2}\right)-Y_{2}+1-Y_{1}\right\} .
$$

Consider a solution

$$
\left(\left(x_{i}\right)_{i \in M},\left(y_{1, i}\right)_{i \in M}, \ldots,\left(y_{6, i}\right)_{i \in M}\right) \text { of } G=0 \text { in } k^{M} .
$$

If $\left(x_{i}\right)_{i \in \mathbb{N}}$ contains only finitely many zeroes, then $\left(y_{1, i}\right)_{i \in \mathbb{N}}$ contains only finitely many nonzero elements. In other words, there exists $N \in \mathbb{N}$ such that $y_{1, i}=0$ for every $i>N$. Thus, $y_{2, i+1}=y_{2, i}-1$ for every $i>N$, so there exists $i_{0}$ such that $y_{2, i_{0}}<0$. This contradicts the fact that $y_{2, i_{0}}=y_{3, i_{0}}^{2}+y_{4, i_{0}}^{2}+y_{5, i_{0}}^{2}+y_{6, i_{0}}^{2} \geqslant 0$.

To prove the second claim of the lemma, consider a sequence $\left(x_{i}\right)_{i \in M}$ such that $\left(x_{i}\right)_{i \in \mathbb{N}}$ contains infinitely many zeroes. We will construct a corresponding solution of $G=0$ in $k^{M}$. Consider positive integers $i_{1}<i_{2}<i_{3}<\cdots$ such that $x_{i_{n}}=0$ for every $n>0$. Then we set

$$
\begin{aligned}
& y_{1, j}=\left\{\begin{array}{ll}
i_{m+1}-i_{m}, & \text { if } j=i_{m} \text { for some } m, \\
0, & \text { otherwise }
\end{array}\right. \text { and } \\
& y_{2, j}= \begin{cases}i_{m+1}-j, & \text { if } i_{m}<j \leqslant i_{m+1} \text { for some } m, \\
i_{1}-j, & \text { otherwise. }\end{cases}
\end{aligned}
$$

The choice of $i_{1}, i_{2}, \ldots$ implies that $x_{j} y_{1, j}=0$ for all $j \in M$. A direct computation shows that $y_{2, j+1}=y_{2, j}-1+y_{1, j}$ for all $j \in M$. Finally, the existence of $y_{3, j}$, $y_{4, j}, y_{5, j}, y_{6, j}$ satisfying $y_{2, j}=y_{3, j}^{2}+y_{4, j}^{2}+y_{5, j}^{2}+y_{6, j}^{2}$ follows from the fact that $y_{2, j}$ is a nonnegative integer and Lagrange's four-square theorem [10, Theorem 369]. 
We return to the proof of Theorem 3.6. We apply Lemma $4.4 n+1$ times, and obtain $n+1$ systems $G_{0}=0, \ldots, G_{n}=0$ with distinguished unknowns $X_{0}, \ldots$, $X_{n}$. We set

$$
\begin{aligned}
F_{P}:= & G_{0} \cup \cdots \cup G_{n} \cup\left\{X_{0}-P\left(X_{1}, \ldots, X_{n}\right),\right. \\
& \left.\left(\sigma\left(X_{1}\right)-X_{1}\right)^{2}-1, \ldots,\left(\sigma\left(X_{n}\right)-X_{n}\right)^{2}-1\right\} .
\end{aligned}
$$

We will show that $F_{P}=0$ has a solution in $k^{M}$ if and only if $P\left(t_{1}, \ldots, t_{n}\right)=0$ has a solution in $\mathbb{Z}$.

Solution of $F_{P}=0 \Longrightarrow$ solution of $P=0$. Consider a solution of $F_{P}$ in $k^{M}$. For every $0 \leqslant m \leqslant n$, we denote the $X_{m}$-coordinate of the solution by $\left(x_{m, i}\right)_{i \in M}$. For every $1 \leqslant m \leqslant n$, the sequence $\left(x_{m, i}\right)_{i \in M}$ contains infinitely many zeroes due to Lemma 4.4; every two consecutive numbers in the sequence differ by one, and thus all the numbers in the sequence are integers. Since $\left(x_{0, i}\right)_{i \in \mathbb{N}}$ contains infinitely many zeroes, the Diophantine equation $P\left(t_{1}, \ldots, t_{n}\right)=0$ has an integer solution.

Solution of $P=0 \Longrightarrow$ solution of $F_{P}=0$. Consider a solution $\left(a_{1}, \ldots, a_{m}\right)$ of $P\left(t_{1}, \ldots, t_{m}\right)=0$ in $\mathbb{Z}^{n}$. Consider sequences $\left(x_{1, i}\right)_{i \in M}, \ldots,\left(x_{n, i}\right)_{i \in M}$ such that

- every two consecutive numbers in the sequences differ by one;

- for every $1 \leqslant m \leqslant n,\left(x_{m, i}\right)_{i=0}^{\infty}$ contains infinitely many zeros;

- $x_{1, i}=a_{1}, \ldots, x_{n, i}=a_{n}$ for infinitely many $i$.

We define $x_{0, i}$ as $P\left(x_{1, i}, \ldots, x_{n, i}\right)$ for every $i \in M$ and observe that $\left(x_{0, i}\right)_{i \in \mathbb{N}}$ contains infinitely many zeroes. The defined sequences satisfy equations

$$
X_{0}-P\left(X_{1}, \ldots, X_{n}\right)=\left(\sigma\left(X_{1}\right)-X_{1}\right)^{2}-1=\cdots=\left(\sigma\left(X_{n}\right)-X_{n}\right)^{2}-1=0 .
$$

The second part of Lemma 4.4 implies that, for every $0 \leqslant m \leqslant n$, the sequence $\left(x_{m, i}\right)_{i \in M}$ can be extended to a solution of $G_{m}=0$. Thus, we obtain a solution of $F_{P}=0$.

4.3. Proofs of Theorem 3.7 and Corollary 3.8. We will first establish a lemma that draws a connection between the strong difference Nullstellensatz and iterations of piecewise polynomial maps. This lemma is crucial for the proof of Theorem 3.7 and for establishing the counterexample in Theorem 3.2.

Let $k$ be a field. For a subset $F$ of $k[\mathbf{X}]=k\left[X_{1}, \ldots, X_{n}\right]$, we denote the closed subset of $\mathbb{A}_{k}^{n}$ defined by $F$ with $V(F)$. Recall that a subset $V$ of $\mathbb{A}_{k}^{n}$ is locally closed if it is of the form $V(F) \backslash V\left(F^{\prime}\right)$ for subsets $F$ and $F^{\prime}$ of $k[\mathbf{X}]$. A regular function $f: V \rightarrow \mathbb{A}_{k}^{1}$ on $V$ is a polynomial function if it is the restriction of a regular function $\mathbb{A}_{k}^{n} \rightarrow \mathbb{A}_{k}^{1}$, that is, if it is given by a polynomial in $k[\mathbf{X}]$. 
DEFINITION 4.5. A piecewise polynomial function $\mathbb{A}_{k}^{n} \rightarrow \mathbb{A}_{k}^{1}$ is a partition of $\mathbb{A}_{k}^{n}$ into locally closed subsets $C_{1}, \ldots, C_{m}$, together with a polynomial function $f_{i}$ on every $C_{i}$.

A piecewise polynomial map $\mathbf{p}: \mathbb{A}_{k}^{n} \rightarrow \mathbb{A}_{k}^{n}$ is an $n$-tuple $\left(p_{1}, \ldots, p_{n}\right)$ of piecewise polynomial functions.

Note that a piecewise polynomial map p: $\mathbb{A}_{k}^{n} \rightarrow \mathbb{A}_{k}^{n}$ defines an actual map $\mathbb{A}_{k}^{n}(K) \rightarrow \mathbb{A}_{k}^{n}(K)$ for every field extension $K$ of $k$.

Lemma 4.6. Let $M$ be $\mathbb{N}$ or $\mathbb{Z}$. Let $\mathbf{p}: \mathbb{A}_{k}^{n} \rightarrow \mathbb{A}_{k}^{n}$ be a piecewise polynomial map and let $V$ be a closed subset of $\mathbb{A}_{k}^{n}$. Then there exist (and can be computed algorithmically) an integer $r \geqslant 1$ and difference polynomials $f_{1}, \ldots, f_{\ell}, g \in$ $k\left[\sigma^{i}\left(T_{1}\right), \ldots, \sigma^{i}\left(T_{r}\right) \mid i \in \mathbb{N}\right]$ such that for every field extension $K$ of $k$, the following two statements are equivalent:

- There exists a sequence $\left(\mathbf{x}_{i}\right)_{i \in \mathbb{N}}=\left(x_{1, i}, \ldots, x_{n, i}\right)_{i \in \mathbb{N}} \in\left(K^{\mathbb{N}}\right)^{n}$ such that

$$
\mathbf{x}_{0} \in V(K), \quad \mathbf{x}_{i+1}=\mathbf{p}\left(\mathbf{x}_{i}\right) \quad \text { for every } i \in \mathbb{N},
$$

and $x_{n, i} \neq 0$ for $i \geqslant 1$.

- There exists a solution of $f_{1}=\cdots=f_{\ell}=0$ in $\left(K^{M}\right)^{r}$ such that $g$ does not vanish on this solution.

Before showing the construction of the systems of difference equations in full generality, we will illustrate it on two examples.

EXAMPLE 4.7. We will use the notation of Lemma 4.6. Let

$$
k=\mathbb{C}, \quad n=1, \quad p(x)=x+1, \quad V=\{0\} .
$$

We introduce two difference variables $X$ and $U^{\prime}$, and consider difference polynomials

$$
\tilde{f}_{1}:=\sigma(X)-p(X)=\sigma(X)-(X+1), \quad \tilde{f_{2}}:=X U^{\prime}-1 .
$$

Every sequence $\left(x_{i}\right)_{i \in \mathbb{N}}$ satisfying $\widetilde{f}_{1}=0$ obeys the recurrence $x_{i+1}=p\left(x_{i}\right)$. Furthermore, such a sequence can be extended to a solution of $\widetilde{f_{1}}=\widetilde{f_{2}}=0$ if and only if $x_{i} \neq 0$ for every $i \geqslant 0$ (compare with $i \geqslant 1$ in the statement of the lemma).

Now we would like to force $\left(x_{i}\right)_{i \in \mathbb{N}}$ to have at least one term in $V$. For doing this, we will introduce one more difference variable $U$ and difference polynomials

$$
f_{3}:=U(U-1), \quad f_{4}:=(\sigma(U)-U)(\sigma(U)-U-1), \quad g:=\sigma(U)-U .
$$


Consider a sequence $\left(u_{i}\right)_{i \in \mathbb{N}}$, which is a solution of $f_{3}=f_{4}=0, g \neq 0$. The equations $f_{3}=f_{4}=0$ imply that $\left(u_{i}\right)_{i \in \mathbb{N}}$ is a 'step sequence' in the sense that it takes only values zero and one and each next value is the same or greater by one. There are three types of sequences that satisfy these conditions:

$$
(0, \ldots, 0,1,1, \ldots), \quad(0,0,0,0, \ldots), \quad(1,1,1,1, \ldots) .
$$

The two last are ruled out by the extra condition $g \neq 0$.

We introduce new polynomials:

$$
\begin{aligned}
& f_{5}:=(\sigma(U)-U) X, \quad f_{1}:=\sigma(U) \widetilde{f_{1}}=\sigma(U)(\sigma(X)-(X+1)), \\
& f_{2}=U \widetilde{f_{2}}=U\left(X U^{\prime}-1\right) .
\end{aligned}
$$

Consider a triple of sequences $\left(x_{i}, u_{i}, u_{i}^{\prime}\right)_{i \in \mathbb{N}}$ satisfying

$$
f_{1}=f_{2}=f_{3}=f_{4}=f_{5}=0, \quad g \neq 0 .
$$

As we have shown, there will be $i_{0} \in \mathbb{N}$ such that

$$
u_{0}=\cdots=u_{i_{0}}=0 \quad \text { and } \quad 1=u_{i_{0}+1}=u_{i_{0}+2}=\cdots .
$$

Equation $f_{5}=0$ ensures that $x_{i_{0}} \in V$. The fact that we have multiplied $\widetilde{f}_{1}$ and $\widetilde{f_{2}}$ by $\sigma(U)$ and $U$, respectively, implies that $\widetilde{f}_{1}$ and $\widetilde{f}_{2}$ have to vanish on the indices $i \geqslant i_{0}$ and $i>i_{0}$, respectively.

To summarize, we see that the sequence $\left(y_{i}\right)_{i \in \mathbb{N}}=\left(x_{i_{0}+i}\right)_{i \in \mathbb{N}}$ satisfies $y_{i+1}=$ $p\left(y_{i}\right), y_{0} \in V$, and $y_{i} \neq 0$ for $i \geqslant 1$. On the other hand, any such sequence can be completed by $u=(0,1,1, \ldots)$ and $u^{\prime}=\left(0,1 / y_{1}, 1 / y_{2}, \ldots\right)$ to a solution of Equation (2).

EXAMPLE 4.8. Now we consider a version of Example 4.7 where $p$ is actually a piecewise polynomial function, not just a polynomial. Let

$$
k=\mathbb{C}, \quad n=1, \quad p(x)=\left\{\begin{array}{ll}
x+1, & \text { if } x \neq 2 \\
1, & \text { if } x=2,
\end{array} \quad V=\{0\} .\right.
$$

We define $C_{1}:=\mathbb{A}^{1} \backslash\{2\}, C_{2}:=\{2\}, q_{1}(x):=x+1$, and $q_{2}(x):=1$ so that $\left.p\right|_{C_{1}}=q_{1}$ and $\left.p\right|_{C_{2}}=q_{2}$. Our strategy would be to define an indicator sequence that will tell us whether $x_{i} \in C_{2}$ or not. For doing this, we introduce two difference variables $Y$ and $Z$ and difference polynomials

$$
f_{6}:=Z(X-2), \quad f_{7}:=Z+Y(X-2)-1 .
$$


Consider any tuple of sequences $\left(x_{i}, y_{i}, z_{i}\right)_{i \in \mathbb{N}}$ satisfying $f_{6}=f_{7}=0$. Whenever $x_{i} \notin C_{2}, f_{6}=0$ implies that $z_{i}=0$. If $x_{i} \in C_{2}$, then $f_{7}=0$ implies that $z_{i}=1$. Thus $z_{i}$ is an indicator for $x_{i} \in C_{2}$. Therefore, we have

$$
p\left(x_{i}\right)=\left(1-z_{i}\right)\left(x_{i}+1\right)+z_{1} \cdot 1 \quad \text { for every } i \in \mathbb{N} .
$$

We can now adapt the system equation (2) from Example 4.7 as follows. We take the same $f_{2}, f_{3}, f_{4}, f_{5}$, but change $f_{1}$ to be

$$
f_{1}:=\sigma(U)(X-(1-Z)(X+1)-Z)
$$

according to equation (3). Then, combining the argument from Example 4.7 and this example, one can see that any sequence $\left(x_{i}\right)_{i \in \mathbb{N}}$ with

$$
x_{0}=0, \quad x_{i+i}=p\left(x_{i}\right), \quad \text { and } \quad x_{i} \neq 0 \text { for } i \geqslant 1
$$

can be extended to a solution of

$$
f_{1}=f_{2}=\cdots=f_{7}=0, \quad g \neq 0 .
$$

On the other hand, for every solution for the above system of difference equations, the $X$-component satisfies equation (4) after removing several first terms.

Proof of Lemma 4.6. Let $\mathbf{p}=\left(p_{1}, \ldots, p_{n}\right)$. Since finite intersections of locally closed subsets are locally closed, we can find a partition $C_{1}, \ldots, C_{m}$ of $\mathbb{A}_{k}^{n}$ that works for every $p_{i}$. For $j=1, \ldots, m$, let $\mathbf{q}_{j}=\left(q_{j, 1}, \ldots, q_{j, n}\right) \in k[\mathbf{X}]^{n}$ be such that $\mathbf{p}(a)=\mathbf{q}_{j}(a)$ for all $a \in C_{j}(K)$ and all field extensions $K$ of $k$.

For every closed subset $W$ of $\mathbb{A}_{k}^{n}$, we define a polynomial system $S_{W}$ as follows. Let $h_{1}, \ldots, h_{t} \in k[\mathbf{X}]$ be polynomials such that $W=V\left(h_{1}, \ldots, h_{t}\right)$. Let $S_{W}=$ $S_{W}(\mathbf{X}, \mathbf{Y}, Z)$ be the system in the variables $\mathbf{X}=\left(X_{1}, \ldots, X_{n}\right), \mathbf{Y}=\left(Y_{1}, \ldots, Y_{t}\right)$ and $Z$ given by

$$
Z h_{1}(\mathbf{X}), \ldots, Z h_{t}(\mathbf{X}), Z+Y_{1} h_{1}(\mathbf{X})+\cdots+Y_{t} h_{t}(\mathbf{X})-1 .
$$

Note that for a field extension $K$ of $k$ and a solution $(\mathbf{x}, \mathbf{y}, z) \in K^{n+t+1}$, we have $z=1$ if $\mathbf{x} \in W$ and $z=0$ if $\mathbf{x} \notin W$. Moreover, for every field extension $K$ of $k$ and $\mathbf{x} \in K^{n}$, there exist $\mathbf{y} \in K^{t}$ and $z \in K$ such that $(\mathbf{x}, \mathbf{y}, z)$ is a solution of $S_{W}$.

Now for every $j=1, \ldots, m$, write $C_{j}=W_{j} \backslash W_{j}^{\prime}$, where $W_{j}, W_{j}^{\prime}$ are closed subsets of $\mathbb{A}_{k}^{n}$ with $W_{j}^{\prime} \subseteq W_{j}$ and consider the systems $S_{j}=S_{W_{j}}=S_{W_{j}}\left(\mathbf{X}, \mathbf{Y}_{j}, Z_{j}\right)$ and $S_{j}^{\prime}=S_{W_{j}^{\prime}}=S_{W_{j}^{\prime}}\left(\mathbf{X}, \mathbf{Y}_{j}^{\prime}, Z_{j}^{\prime}\right)$. Let $g_{1}, \ldots, g_{s} \in k[\mathbf{X}]$ be such that $V\left(g_{1}, \ldots\right.$, $\left.g_{s}\right)=V$.

Let $S$ denote the system of difference equations in the variables

$$
U, U^{\prime}, \mathbf{X}, \mathbf{Y}_{1}, \ldots, \mathbf{Y}_{m}, Z_{1}, \ldots, Z_{m}, \mathbf{Y}_{1}^{\prime}, \ldots, \mathbf{Y}_{m}^{\prime}, Z_{1}^{\prime}, \ldots, Z_{m}^{\prime}
$$


given by

$$
\begin{gathered}
S_{1}\left(\mathbf{X}, \mathbf{Y}_{1}, Z_{1}\right), \ldots, S_{m}\left(\mathbf{X}, \mathbf{Y}_{m}, Z_{m}\right), S_{1}^{\prime}\left(\mathbf{X}, \mathbf{Y}_{1}^{\prime}, Z_{1}^{\prime}\right), \ldots, S_{m}\left(\mathbf{X}, \mathbf{Y}_{m}^{\prime}, Z_{m}^{\prime}\right), \\
\sigma(U)\left(\sigma(\mathbf{X})-\left(\mathbf{q}_{1}(\mathbf{X})\left(Z_{1}-Z_{1}^{\prime}\right)+\cdots+\mathbf{q}_{m}(\mathbf{X})\left(Z_{m}-Z_{m}^{\prime}\right)\right)\right), \\
U(U-1),(\sigma(U)-U)(\sigma(U)-U-1), \\
U\left(X_{n} U^{\prime}-1\right),(\sigma(U)-U) g_{1}(\mathbf{X}), \ldots,(\sigma(U)-U) g_{s}(\mathbf{X}) .
\end{gathered}
$$

We will show that $S=\left\{f_{1}, \ldots, f_{\ell}\right\}$ and $g=\sigma(U)-U$ have the property of the lemma. To this end, let us fix a field extension $K$ of $k$ and let us first assume that

$$
a=\left(u_{i}, u_{i}^{\prime}, \mathbf{x}_{i}, \mathbf{y}_{1, i}, \ldots, \mathbf{y}_{m, i}, z_{1, i}, \ldots, z_{m, i}, \mathbf{y}_{1, i}^{\prime}, \ldots, \mathbf{y}_{m, i}^{\prime}, z_{1, i}^{\prime}, \ldots, z_{m, i}^{\prime}\right)_{i \in M} \in\left(K^{M}\right)^{r}
$$

is a solution of $S$ such that $\sigma(U)-U$ does not vanish on $a$. We observe that the equations $U(U-1)=0$ and $(\sigma(U)-U)(\sigma(U)-U-1)=0$ imply that either $u_{i}=0$ for all $i, u_{i}=1$ for all $i$ or, there exists an $i_{0} \in M$ such that

$$
u_{i}= \begin{cases}0 & \text { for } i \leqslant i_{0} \\ 1 & \text { for } i>i_{0}\end{cases}
$$

Since $\sigma(U)-U$ does not vanish on $a$, the sequence $\left(u_{i}\right)_{i \in M}$ is of the latter kind. The equations $(\sigma(U)-U) g_{1}(\mathbf{X})=\cdots=(\sigma(U)-U) g_{s}(\mathbf{X})=0$ imply that $g_{1}\left(\mathbf{x}_{i_{0}}\right)=\cdots=g_{s}\left(\mathbf{x}_{i_{0}}\right)=0$, that is, $\mathbf{x}_{i_{0}} \in V(K)$.

For every $j=1, \ldots, m$ and $i \in M$, we have

$$
z_{j, i}= \begin{cases}1 & \text { if } \mathbf{x}_{i} \in W_{j}(K), \\ 0 & \text { if } \mathbf{x}_{i} \notin W_{j}(K) .\end{cases}
$$

Similarly,

$$
z_{j, i}^{\prime}= \begin{cases}1 & \text { if } \mathbf{x}_{i} \in W_{j}^{\prime}(K), \\ 0 & \text { if } \mathbf{x}_{i} \notin W_{j}^{\prime}(K) .\end{cases}
$$

Therefore

$$
z_{j, i}-z_{j, i}^{\prime}= \begin{cases}1 & \text { if } \mathbf{x}_{i} \in C_{j}(K), \\ 0 & \text { if } \mathbf{x}_{i} \notin C_{j}(K) .\end{cases}
$$

Thus the equations $\sigma(U)\left(\sigma(\mathbf{X})-\left(\mathbf{q}_{1}(\mathbf{X})\left(Z_{1}-Z_{1}^{\prime}\right)+\cdots+\mathbf{q}_{m}(\mathbf{X})\left(Z_{m}-Z_{m}^{\prime}\right)\right)\right)=0$ show that $\mathbf{x}_{i+1}=\mathbf{p}\left(\mathbf{x}_{i}\right)$ for all $i \geqslant i_{0}$. Finally, the equation $U\left(U^{\prime} X_{n}-1\right)=0$ shows that $x_{n, i} \neq 0$ for $i>i_{0}$. Therefore the sequence $\left(\mathbf{x}_{i_{0}+i}\right)_{i \in \mathbb{N}}$ has the desired properties. 
Conversely, let us assume that the sequence $\left(\mathbf{x}_{i}\right)_{i \in \mathbb{N}}$ satisfies $\mathbf{x}_{0} \in V(K), \mathbf{x}_{i+1}=$ $\mathbf{p}\left(\mathbf{x}_{i}\right)$ for $i \in \mathbb{N}$ and $x_{n, i} \neq 0$ for $i \geqslant 1$. We extend this sequence to a solution

$$
a=\left(u_{i}, u_{i}^{\prime}, \mathbf{x}_{i}, \mathbf{y}_{1, i}, \ldots, \mathbf{y}_{m, i}, z_{1, i}, \ldots, z_{m, i}, \mathbf{y}_{1, i}^{\prime}, \ldots, \mathbf{y}_{m, i}^{\prime}, z_{1, i}^{\prime}, \ldots, z_{m, i}^{\prime}\right)_{i \in M} \in\left(K^{M}\right)^{r}
$$

of $S$ such that $g$ does not vanish at $a$. For $M=\mathbb{Z}$, we set $x_{j, i}=0$ for $i<0$ and $j=1, \ldots, m$. We define

$$
u_{i}=\left\{\begin{array}{ll}
1 & \text { for } i \geqslant 1, \\
0 & \text { otherwise }
\end{array} \quad \text { and } \quad u_{i}^{\prime}= \begin{cases}\frac{1}{x_{n, i}} & \text { for } i \geqslant 1 \\
0 & \text { otherwise }\end{cases}\right.
$$

For $i \in M$, we choose $\mathbf{y}_{j, i} \in K^{s_{j}}$ and $z_{j, i} \in K$ such that $\left(\mathbf{x}_{i}, \mathbf{y}_{j, i}, z_{j, i}\right)$ is a solution of $S_{j}\left(\mathbf{X}, \mathbf{Y}_{j}, Z_{j}\right)$. Similarly, we choose $\mathbf{y}_{j, i}^{\prime} \in K^{s_{j}^{\prime}}$ and $z_{j, i}^{\prime} \in K$ such that $\left(\mathbf{x}_{i}, \mathbf{y}_{j, i}^{\prime}\right.$, $\left.z_{j, i}^{\prime}\right)$ is a solution of $S_{j}^{\prime}\left(\mathbf{X}, \mathbf{Y}_{j}^{\prime}, Z_{j}^{\prime}\right)$. Then $a$ is a solution of $S$ such that $g$ does not vanish at $a$.

We will need one more preparatory lemma for the proof of Theorem 3.7. For every $n$, by $T_{n}$ we will denote the sequence of all nondecreasing $n$-tuples of nonnegative integers listed in ascending colexicographic order. For example,

$$
\begin{aligned}
& T_{1}=((0),(1),(2),(3), \ldots) \text { and } \\
& T_{2}=((0,0),(0,1),(1,1),(0,2),(1,2),(2,2), \ldots) .
\end{aligned}
$$

LEMMA 4.9. For every $n \geqslant 1$, there exists a piecewise polynomial map $p: \mathbb{A}_{\mathbb{Q}}^{n} \rightarrow$ $\mathbb{A}_{\mathbb{Q}}^{n}$ such that for the sequence $\left(\boldsymbol{x}_{i}\right)_{i \in \mathbb{N}}=\left(x_{1, i}, \ldots, x_{n, i}\right)_{i \in \mathbb{N}}$ defined by

$$
\mathbf{x}_{0}=(0, \ldots, 0) \quad \& \quad \mathbf{x}_{i+1}=\mathbf{p}\left(\mathbf{x}_{i}\right) \text { for all } i \in \mathbb{N},
$$

we have $\left(\mathbf{x}_{i}\right)_{i \in \mathbb{N}}=T_{n}$.

Proof. The successor of a nondecreasing $n$-tuple $\left(a_{1}, \ldots, a_{n}\right) \in \mathbb{N}^{n}$ in $T_{n}$ is $\left(a_{1}\right.$, $\left.\ldots, a_{r-1}, a_{r}+1, a_{r+1}, \ldots, a_{n}\right)$ if there exists an $r$ with $1 \leqslant r<n$ such that $a_{1}=\cdots=a_{r} \neq a_{r+1}$ and $\left(0, \ldots, 0, a_{n}+1\right)$ if there exists no such $r$, that is, if $a_{1}=\cdots=a_{n}$. Thus, the piecewise polynomial map $\mathbf{p}=\left(p_{1}, \ldots, p_{n}\right)$ defined by

$$
p_{i}\left(x_{1}, \ldots, x_{n}\right)= \begin{cases}x_{i}+1 & \text { if } x_{1}=\cdots=x_{i} \neq x_{i+1}, \\ 0 & \text { if } x_{1}=\cdots=x_{n}, \\ x_{i} & \text { otherwise }\end{cases}
$$

for $i=1, \ldots, n-1$ and

$$
p_{n}\left(x_{1}, \ldots, x_{n}\right)= \begin{cases}x_{n}+1 & \text { if } x_{1}=\cdots=x_{n} \\ x_{n} & \text { otherwise }\end{cases}
$$


has the desired property.

Proof of Theorem 3.7. We will prove Theorem 3.7 by showing that the decidability of the problem of Theorem 3.7 implies the decidability of Hilbert's tenth problem for the integers. Let $P \in \mathbb{Z}\left[t_{1}, \ldots, t_{n}\right]$ with $P(0, \ldots, 0) \neq 0$ and consider the piecewise polynomial map $\mathbf{q}: \mathbb{A}_{\mathbb{Q}}^{m} \rightarrow \mathbb{A}_{\mathbb{Q}}^{m}$, where $m=n \cdot n !+1$, defined as follows: Thinking of $\mathbb{A}_{\mathbb{Q}}^{m}$ as $\left(\prod_{\pi \in S_{n}} \mathbb{A}_{\mathbb{Q}}^{n}\right) \times \mathbb{A}_{\mathbb{Q}}^{1}$, we write $\boldsymbol{x}=\left(\left(\boldsymbol{x}_{\pi}\right)_{\pi \in S_{n}}\right.$, $x_{r}$ ), where each $\boldsymbol{x}_{\pi}$ is an $n$-tuple. We set

$$
\mathbf{q}(\boldsymbol{x})=\left(\left(\mathbf{p}_{\pi}\left(x_{\pi}\right)\right)_{\pi \in S_{n}}, \prod_{\pi \in S_{n}} P\left(\boldsymbol{x}_{\pi}\right)\right),
$$

where $\mathbf{p}_{\pi}: \mathbb{A}_{\mathbb{Q}}^{n} \rightarrow \mathbb{A}_{\mathbb{Q}}^{n}$ is the map p: $\mathbb{A}_{\mathbb{Q}}^{n} \rightarrow \mathbb{A}_{\mathbb{Q}}^{n}$ from Lemma 4.9 but conjugated with the permutation $\pi$. So, if we define $\left(\boldsymbol{x}_{i}\right)_{i \in \mathbb{N}} \in\left(\mathbb{Q}^{\mathbb{N}}\right)^{m}$ by $\boldsymbol{x}_{0}=(0, \ldots, 0)$ and $\boldsymbol{x}_{i+1}=\mathbf{q}\left(\boldsymbol{x}_{i}\right)$ for $i \geqslant 0$, we see that, for every element $a$ of $\mathbb{N}^{n}$, there exist $i \in \mathbb{N}$ and $\pi \in S_{n}$ such that $\left(\boldsymbol{x}_{i}\right)_{\pi}=a$. It follows that $x_{r, i} \neq 0$ for every $i \geqslant 1$ if and only if $P$ has no solution in $\mathbb{N}^{n}$. Thus, by Lemma 4.6, there exist an integer $r \geqslant 1$ and difference polynomials

$$
f_{1}, \ldots, f_{\ell}, g \in \mathbb{Q}\left[\sigma^{i}\left(T_{1}\right), \ldots, \sigma^{i}\left(T_{r}\right) \mid i \in \mathbb{N}\right] \subseteq k_{0}\left[\sigma^{i}\left(T_{1}\right), \ldots, \sigma^{i}\left(T_{r}\right) \mid i \in \mathbb{N}\right]
$$

such that $g$ does not vanish on every solution of $f_{1}=\cdots=f_{\ell}=0$ in $k^{M}$ if and only if $P$ has no solution in $\mathbb{N}^{n}$.

Proof of Corollary 3.8. The undecidability of (P1) follows from Theorem 3.7 and the fact that the system $f_{1}=\cdots=f_{\ell}=0, g \neq 0$ has a solution in $k^{M}$ if and only if $g=0$ does not hold for some solution of $f_{1}=\cdots=f_{\ell}=0$ in $k^{M}$.

Let $K$ be an uncountable algebraically closed field containing $k$. Theorem 3.1 implies that

$$
g \in \sqrt{\left\langle\sigma^{m}\left(f_{1}\right), \ldots, \sigma^{m}\left(f_{\ell}\right) \mid m \in M\right\rangle}
$$

if and only if $g=0$ vanishes on every solution of $f_{1}=\cdots=f_{\ell}=0$ in $K^{M}$. Thus, the undecidability of (P2) follows from Theorem 3.7.

4.4. Proof of Proposition 3.9. We will first consider the case $M=\mathbb{Z}^{2}$ and then reduce the case $M=\mathbb{N}^{2}$ to it.

Consider a set $\mathcal{D}=\left\{D_{1}, \ldots, D_{n}\right\}$ of dominoes (in the sense of [1, p. 1]) such that the labels on the edges are integers from 1 to $N$. We will construct a finite set $F \subset \mathbb{Q}\left[\sigma^{m}(X), \sigma^{m}(Y) \mid m \in \mathbb{Z}^{2}\right]$ such that the tilings of the plane by $\mathcal{D}$ correspond bijectively to the solutions of $F=0$ in $k^{\mathbb{Z}^{2}}$. 
For every $1 \leqslant i \leqslant n$, by $D_{i}(l), D_{i}(r), D_{i}(t)$, and $D_{i}(b)$ we denote the marks on the left, right, top, and bottom edges of $D_{i}$, respectively. Let

$$
\begin{aligned}
F:= & \{(X-1)(X-2) \cdots(X-N),(Y-1)(Y-2) \cdots(Y-N), \\
& \prod_{k=1}^{n}\left(\left(D_{k}(b)-X\right)^{2}+\left(D_{k}(t)-\sigma^{(0,1)}(X)\right)^{2}\right. \\
& \left.\left.+\left(D_{k}(l)-Y\right)^{2}+\left(D_{k}(r)-\sigma^{(1,0)}(Y)\right)^{2}\right)\right\} .
\end{aligned}
$$

Consider any tiling of the plane by dominoes from $\mathcal{D}$. For every $i, j \in \mathbb{Z}$, we denote

- the mark on the edge connecting the points $(i, j)$ and $(i+1, j)$ by $x_{i, j}$;

- the mark on the edge connecting the points $(i, j)$ and $(i, j+1)$ by $y_{i, j}$.

Then $\left(\left(x_{i, j}\right)_{i, j \in \mathbb{Z}},\left(y_{i, j}\right)_{i, j \in \mathbb{Z}}\right)$ is a solution of $F=0$ in $k^{\mathbb{Z}^{2}}$ because

- all marks are integers from 1 to $N$, so the first two polynomials in $F$ vanish; and

- the last polynomial in $F$ vanishes if and only if each square is covered by a domino from $\mathcal{D}$.

For the other direction, let $\left(\left(x_{i, j}\right)_{i, j \in \mathbb{Z}},\left(y_{i, j}\right)_{i, j \in \mathbb{Z}}\right)$ be a solution of $F=0$ in $k^{\mathbb{Z}^{2}}$. Then all $x_{i, j}$ 's and $y_{i, j}$ 's are integers from 1 to $N$, so they are valid edge marks. Moreover, if we mark the edges of the integer lattice by numbers $x_{i, j}$ and $y_{i, j}$ as described above, then the fact that $\left(\left(x_{i, j}\right)_{i, j \in \mathbb{Z}},\left(y_{i, j}\right)_{i, j \in \mathbb{Z}}\right)$ satisfies the last equation in $F=0$ implies that these marks produce a tiling by dominoes from $\mathcal{D}$.

Since the problem of determining whether there is a tiling of the plane by a given set of dominoes is undecidable [1, page 2], the problem of determining consistency of a system of $\mathbb{Z}^{2}$-polynomials in $k^{\mathbb{Z}^{2}}$ is also undecidable.

The undecidability of the consistency problem for $M=\mathbb{N}^{2}$ follows from the above argument and the following lemma.

LEMMA 4.10. Consider $F \subset \mathbb{Q}\left[\sigma^{m}(X), \sigma^{m}(Y) \mid m \in \mathbb{N}^{2}\right]$ defined by equation (5). Then $F=0$ has a solution in $k^{\mathbb{Z}^{2}}$ if and only if it has a solution in $k^{\mathbb{N}^{2}}$.

Proof. Consider a solution of $F=0$ in $k^{\mathbb{Z}^{2}}$. If we restrict it on $\mathbb{N}^{2}$, we will obtain a solution of $F=0$ in $k^{\mathbb{N}^{2}}$. 
Assume that $F=0$ does not have a solution in $k^{\mathbb{Z}^{2}}$. Let $K$ be an uncountable algebraically closed field containing $k$. The first two equations of $F=0$ force all the coordinates of any solution of $F=0$ in $K$ to be integers from 1 to $N$. Thus, $F=0$ does not have a solution in $K^{\mathbb{Z}^{2}}$ as well. Then Theorem 3.1 implies that 1 belongs to the $\mathbb{Z}^{2}$-invariant ideal generated by $F=\left\{f_{1}, f_{2}, f_{3}\right\}$, that is, there exists a positive integer $H$ such that

$$
1=\sum_{\ell=1}^{3}\left(\sum_{-H \leqslant i, j \leqslant H} c_{i, j} \sigma^{(i, j)}\left(f_{\ell}\right)\right),
$$

where $c_{i, j} \in K\left[\sigma^{m}(X), \sigma^{m}(Y) \mid m \in \mathbb{Z}^{2}\right]$ and $-H \leqslant a, b \leqslant H$ for every $\sigma^{(a, b)}$ appearing in $c_{i, j}$. Acting by $\sigma^{(H, H)}$ on equation (6), we conclude that 1 belongs to the $\mathbb{N}^{2}$-invariant ideal generated by $F$ in $K\left[\sigma^{m}(X), \sigma^{m}(Y) \mid m \in \mathbb{N}^{2}\right]$. Thus, $F=0$ does not have solutions in $k^{\mathbb{N}^{2}}$.

4.5. Proof of Proposition 3.10. We will prove Proposition 3.10 by reducing to Corollary 3.8. More precisely, for every set of difference polynomials $f_{1}, \ldots, f_{\ell}$, $g \in k_{0}\left[\sigma^{i}(\mathbf{X}) \mid i \in \mathbb{N}\right]$ with $\mathbf{X}=\left(X_{1}, \ldots, X_{n}\right)$, we will construct a system $F=0$ of $M_{2}$-polynomials over $k_{0}$ such that there exists a solution of $f_{1}=\cdots=f_{\ell}=0$, $g \neq 0$ in $k^{\mathbb{N}}$ if and only if $F=0$ has a solution in $k^{M_{2}}$.

By adding new variables and equations, we may assume that $g \in k_{0}[\mathbf{X}]$ and $f_{1}, \ldots, f_{\ell} \in k_{0}[\mathbf{X}, \sigma(\mathbf{X})]$. Let $\mathbf{Y}=\left(Y_{1}, \ldots, Y_{n}\right)$, and denote the generators of $M_{2}$ by $a$ and $b$. From $f_{1}, \ldots, f_{\ell}, g$, we obtain

$$
\tilde{f}_{1}, \ldots, \tilde{f}_{\ell}, \tilde{g} \in k_{0}\left[\sigma^{m}(\mathbf{Y}), \sigma^{m}(Z) \mid m \in M_{2}\right]
$$

by replacing every $\sigma$ by $\sigma^{a}$ and every $X_{i}$ by $Y_{i}$. Then we set

$$
F:=\left\{\tilde{f}_{1}, \ldots, \tilde{f}_{\ell}, Z \sigma^{b}(\tilde{g})-1\right\}
$$

Let $\left(\boldsymbol{y}_{m}, z_{m}\right)_{m \in M_{2}}$ be a solution of $F=0$ in $k^{M_{2}}$. Then $\tilde{f}_{1}=\cdots=\tilde{f}_{\ell}=0$ implies that $\left\{\mathbf{y}_{b a^{i}}\right\}_{i \in \mathbb{N}}$ is a solution of $f_{1}=\cdots=f_{\ell}=0$ in $k^{\mathbb{N}}$. Furthermore, the equation $Z \sigma^{b}(\tilde{g})-1=0$ implies that $g\left(\boldsymbol{y}_{b}\right) \neq 0$, so $g$ does not vanish on this solution.

Conversely, let $\left(\mathbf{x}_{i}\right)_{i \in \mathbb{N}}$ be a solution of $f_{1}=\cdots=f_{\ell}=0, g \neq 0$. By applying $\sigma$ to it, we may further assume that $c:=g\left(\mathbf{x}_{0}\right) \neq 0$. For every $m \in M_{2}$, we denote by $A(m)$ the largest $i \in \mathbb{N}$ such that $m$ can be written as $m^{\prime} a^{i}$ for some $m^{\prime} \in M_{2}$. For every $m \in M_{2}$, we define $\mathbf{y}_{m}:=\mathbf{x}_{A(m)}$ and $z_{m}:=c^{-1}$. We claim that 
$\left(\mathbf{y}_{m}, z_{m}\right)_{m \in M_{2}}$ is a solution of $F=0$. Let $\mathbf{t}_{0}$ and $\mathbf{t}_{1}$ be $n$-tuples of new algebraic indeterminates. For every $1 \leqslant i \leqslant \ell$, let $P_{i} \in k_{0}\left[\mathbf{t}_{0}, \mathbf{t}_{1}\right]$ be a polynomial such that $f_{i}(\mathbf{X})=P_{i}(\mathbf{X}, \sigma(\mathbf{X}))$. Then $\tilde{f}_{i}(\mathbf{Y})=P_{i}\left(\mathbf{Y}, \sigma^{a}(\mathbf{Y})\right)$. For every $m_{0} \in M_{2}$, we have

$$
\begin{aligned}
\tilde{f}_{i}\left(\left(\mathbf{y}_{m}\right)_{m \in M_{2}}\right)_{m_{0}} & =P_{i}\left(\mathbf{y}_{m_{0}}, \mathbf{y}_{m_{0} a}\right)=P_{i}\left(\mathbf{x}_{A\left(m_{0}\right)}, \mathbf{x}_{A\left(m_{0} a\right)}\right) \\
& =P_{i}\left(\mathbf{x}_{A\left(m_{0}\right)}, \mathbf{x}_{A\left(m_{0}\right)+1}\right)=f_{i}\left(\left(\mathbf{x}_{i}\right)_{i \in \mathbb{N}}\right)_{A\left(m_{0}\right)}=0 .
\end{aligned}
$$

Let $Q \in k_{0}\left[\mathbf{t}_{0}\right]$ be a polynomial such that $g(\mathbf{X})=Q(\mathbf{X})$ and $\tilde{g}(\mathbf{Y})=Q(\mathbf{Y})$. Then, for every $m_{0} \in M_{2}$, we also have

$$
\sigma^{b}\left(\tilde{g}\left(\left(\mathbf{y}_{m}\right)_{m \in M_{2}}\right)\right)_{m_{0}}=Q\left(\mathbf{y}_{m_{0} b}\right)=Q\left(\mathbf{x}_{0}\right)=g\left(\mathbf{x}_{0}\right)=c .
$$

This proves the claim.

4.6. Proof of Theorem 3.2. In this section, we present an example that shows that the assumption $|k|>|M|$ cannot be omitted from Theorem 3.1. In more detail, we present a finite system $F \subseteq \overline{\mathbb{Q}}\left[\sigma^{i}\left(T_{1}\right), \ldots, \sigma^{i}\left(T_{r}\right) \mid i \in \mathbb{N}\right]$ of difference polynomials (with respect to $M=\mathbb{N}$ ) such that $\mathcal{I}(\mathcal{V}(F)) \supsetneqq \sqrt{\left\langle\sigma^{i}(F) \mid i \in \mathbb{N}\right\rangle}$.

Before going into the details of the construction of $F$, we explain the underlying ideas. Very roughly, the idea is to construct a piecewise polynomial map $\mathbf{p}: \mathbb{A}_{\mathbb{Q}}^{n} \rightarrow$ $\mathbb{A}_{\mathbb{Q}}^{n}$ that can detect if a given number is algebraic or transcendental and then to obtain $F$ from $\mathbf{p}$ via Lemma 4.6. More precisely, we will proceed in the following steps:

(a) Construct a piecewise polynomial map $\mathbf{p}: \mathbb{A}_{\mathbb{Q}}^{n} \rightarrow \mathbb{A}_{\mathbb{Q}}^{n}$ such that for $\mathbf{x}_{0}=(c$, $0, \ldots, 0,1) \in \mathbb{C}^{n}$ and $\mathbf{x}_{i+1}=\mathbf{p}\left(x_{i}\right)$, we have the following property:

$$
\text { the sequence }\left(x_{n, i}\right)_{i \in \mathbb{N}} \text { contains } 0 \Longleftrightarrow c \in \overline{\mathbb{Q}} \text {. }
$$

(b) Apply Lemma 4.6 with $V=\mathbb{A}_{\mathbb{Q}}^{1} \times\{0\} \times \cdots \times\{0\} \times\{1\} \subseteq \mathbb{A}_{\mathbb{Q}}^{n}$ and $\mathbf{p}$ being the map constructed in the previous step. This gives rise to difference polynomials $f_{1}, \ldots, f_{\ell}, g \in \mathbb{Q}\left[\sigma^{i}\left(T_{1}\right), \ldots, \sigma^{i}\left(T_{r}\right) \mid i \in \mathbb{N}\right]$ such that for every field extension $K$ of $\mathbb{Q}$, the following are equivalent:

- $g$ vanishes on every solution of $f_{1}=\cdots=f_{\ell}=0$ in $\left(K^{\mathbb{N}}\right)^{r}$;

- $K \subseteq \overline{\mathbb{Q}}$.

(c) Taking $K=\overline{\mathbb{Q}}$, we see that $g \in \mathcal{I}(\mathcal{V}(F))$. On the other hand, since there is a solution of $f_{1}=\cdots=f_{\ell}=0$ in $\left(\mathbb{C}^{\mathbb{N}}\right)^{r}$, on which $g$ does not vanish, we conclude that $g \notin \sqrt{\left\langle\sigma^{i}(F) \mid i \in \mathbb{N}\right\rangle}$. 
The piecewise polynomial map p: $\mathbb{A}_{\mathbb{Q}}^{n} \rightarrow \mathbb{A}_{\mathbb{Q}}^{n}$ is explicitly given below (indeed we will see that one can choose $n=5$ ) and the proof of Lemma 4.6 is constructive. So, in principle it would be possible to explicitly determine $r$,

$$
F=\left\{f_{1}, \ldots, f_{\ell}\right\} \subseteq \overline{\mathbb{Q}}\left[\sigma^{i}\left(T_{1}\right), \ldots, \sigma^{i}\left(T_{r}\right) \mid i \in \mathbb{N}\right]
$$

and $g \in \mathcal{I}(\mathcal{V}(F)) \backslash \sqrt{\left\langle\sigma^{i}(F) \mid i \in \mathbb{N}\right\rangle}$. However, since the piecewise polynomial map p: $\mathbb{A}_{\mathbb{Q}}^{5} \rightarrow \mathbb{A}_{\mathbb{Q}}^{5}$ is already fairly complicated, this would be a very tedious task, yielding an enormously large system $F$. Moreover, we do not expect any deeper insight from determining $F$ explicitly.

We will next define the piecewise polynomial map $\mathbf{p}: \mathbb{A}_{\mathbb{Q}}^{5} \rightarrow \mathbb{A}_{\mathbb{Q}}^{5}$ that detects whether or not a given number is algebraic. Again, we first explain the underlying idea. The piecewise polynomial map p: $\mathbb{A}_{\mathbb{Q}}^{5} \rightarrow \mathbb{A}_{\mathbb{Q}}^{5}$ should have the following property: If $K$ is a field extension of $\mathbb{Q}, \mathbf{x}_{0}=(c, 0,0,0,1) \in K^{5}$ and $\mathbf{x}_{i+1}=\mathbf{p}\left(x_{i}\right)$, then $\left(x_{5, i}\right)_{i \in \mathbb{N}}$ contains 0 if and only if $c$ is algebraic. This property will be satisfied if the sequence $x_{5, i}$ consists of all expressions of the form $P(c)$, where $P$ ranges over all nonzero polynomials in $\mathbb{Z}[x]$. To achieve the latter, we will generate all elements of $\mathbb{Z}[x]$ under iteration. We use the observation that, up to multiplication with \pm 1 , every element of $\mathbb{Z}[x]$ can be obtained from 1 by iterating the following three operations (in a specific order): $P \mapsto P+1, P \mapsto x P, P \mapsto-x P$. We formulate a more precise statement in Lemma 7.

We set $P_{\varnothing}(x)=1$, and for $a=\left(a_{m}, \ldots, a_{0}\right) \in\{0,1,2\}^{m+1}$, we define $P_{a}(x) \in$ $\mathbb{Z}[x]$ recursively by

$$
P_{a}(x)= \begin{cases}x P_{a^{\prime}}(x) & \text { if } a_{m}=0, \\ -x P_{a^{\prime}}(x) & \text { if } a_{m}=1, \\ P_{a^{\prime}}(x)+1 & \text { if } a_{m}=2,\end{cases}
$$

where $a^{\prime}=\left(a_{m-1}, \ldots, a_{0}\right)$ (if $\left.m=0, a^{\prime}=\varnothing\right)$. For $N \in \mathbb{N}$ with base 3 expansion

$$
N=a_{m} 3^{m}+a_{m-1} 3^{m-1}+\cdots+a_{0},
$$

that is, $a_{0}, \ldots, a_{m} \in\{0,1,2\}$ and $a_{m} \neq 0$, we set $P_{N}(x)=P_{a}(x)$ for $a=\left(a_{m}\right.$, $\left.\ldots, a_{0}\right)$. For $N=0$, we set $P_{N}(x)=P_{\varnothing}(x)=1$.

LEMMA 4.11. For every nonzero polynomial $q(x) \in \mathbb{Z}[x]$, there exists an integer $N \geqslant 0$ such that $P_{N}(x)$ is equal to $q(x)$ or $-q(x)$.

Proof. The set of polynomials in $\mathbb{Z}[x]$ that can be obtained from 1 by a finite sequence of the three operations $P(x) \mapsto x P(x), P(x) \mapsto-x P(x)$, and $P(x) \mapsto$ $P(x)+1$ is the set of nonzero polynomials in $\mathbb{Z}[x]$ whose constant coefficient is 
nonnegative. Thus, up to multiplication with \pm 1 , every nonzero polynomial in $\mathbb{Z}[x]$ can be obtained in this way.

The set of all $P_{N}(x)$ 's consists of all polynomials in $\mathbb{Z}[x]$ that can be obtained from 1 by a finite sequence of the three operations $P(x) \mapsto x P(x), P(x) \mapsto$ $-x P(x)$, and $P(x) \mapsto P(x)+1$ under the additional assumption that the last operation is not $x \mapsto x P(x)$. This extra condition comes from the fact that in the base 3 expansion $N=a_{m} 3^{m}+a_{m-1} 3^{m-1}+\cdots+a_{0}$ of $N$ one necessarily has $a_{m} \neq 0$.

Let $q(x) \in \mathbb{Z}[x]$ be a nonzero polynomial. Multiplying $q(x)$ with -1 if necessary, we may assume that the constant coefficient of $q(x)$ is nonnegative. Thus, as observed above, $q(x)=P_{a}(x)$ for a suitable tuple

$$
a=\left(a_{m}, \ldots, a_{0}\right) \in\{0,1,2\}^{m+1} .
$$

If $a_{m} \neq 0$, then $q(x)=P_{a}(x)=P_{N}(x)$ for $N=a_{m} 3^{m}+a_{m-1} 3^{m-1}+\cdots+a_{0}$. If $a_{m}=0$, then $q(x)=-P_{\widetilde{a}}(x)=-P_{\widetilde{N}}(x)$ for $\widetilde{a}=\left(1, a_{m-1}, \ldots, a_{0}\right)$ and

$$
\widetilde{N}=1 \cdot 3^{m}+a_{m-1} 3^{m-1}+\cdots+a_{0} .
$$

Now that we know how to iteratively produce all nonzero polynomials of $\mathbb{Z}[x]$, at least up to sign, we return to the definition of the piecewise polynomial map p: $\mathbb{A}_{\mathbb{Q}}^{5} \rightarrow \mathbb{A}_{\mathbb{Q}}^{5}$ that should detect whether or not a given number $c$ is algebraic. The idea to produce all the $P_{N}(c)$ 's as the entries of the sequence $x_{5, i}$ is to have one coordinate, say the second coordinate, loop through all the natural numbers $N$, while two other coordinates, say the third and fourth coordinates, are used to compute the base 3 expansion of $N$. This base 3 expansion is then used to create $P_{N}(c)$ in the fifth coordinate according to the rule from (7).

The computation of the base 3 expansion of a given natural number $N$ in the second coordinate works as follows. The fourth coordinate starts looping from 0 , with increments of 1 , until it reaches a natural number $A_{1}$ with the property that $N-3 A_{1} \in\{0,1,2\}$. In other words, $N-3 A_{1}=a_{0}$, where

$$
N=a_{m} 3^{m}+a_{m-1} 3^{m-1}+\cdots+a_{0}
$$

is the base 3 expansion of $N$. Then $A_{1}$ is stored in the third coordinate and the fourth coordinate starts looping again from 0 , with increments of 1 , until it reaches a natural number $A_{2}$ with the property that $A_{1}-3 A_{2} \in\{0,1,2\}$, that is, $A_{1}-3 A_{2}=$ $a_{1}$. Then $A_{2}$ is stored in the third coordinate and the process continues like this until we reach the index $m$, such that $A_{m} \in\{0,1,2\}$, that is, $A_{m}=a_{m}$. At this point, the full base 3 expansion of $N$ has been computed and we start over with $N$ replaced by $N+1$. 
Explicitly, the piecewise polynomial map $\mathbf{p}: \mathbb{A}_{\mathbb{Q}}^{5} \rightarrow \mathbb{A}_{\mathbb{Q}}^{5}$ is defined as $\mathbf{p}=(C$, $N, R, A, P)$, where $Q(x):=x(x-1)(x-2)$ and

$$
\begin{aligned}
& C(\mathbf{x})=x_{1}, \\
& N(\mathbf{x})= \begin{cases}x_{2}+1, & \text { if } x_{3}=0, \\
x_{2}, & \text { if } x_{3} \neq 0,\end{cases} \\
& R(\mathbf{x})= \begin{cases}x_{2}+1, & \text { if } x_{3}=0, \\
x_{3}, & \text { if } x_{3} \neq 0 \& Q\left(x_{3}-3 x_{4}\right) \neq 0, \\
x_{4}, & \text { if } x_{3} \neq 0 \& Q\left(x_{3}-3 x_{4}\right)=0,\end{cases} \\
& P(\mathbf{x})= \begin{cases}0, & \text { if } x_{3}=0, \\
x_{4}+1, & \text { if } x_{3} \neq 0 \& Q\left(x_{3}-3 x_{4}\right) \neq 0, \\
0, & \text { if } x_{3} \neq 0 \& Q\left(x_{3}-3 x_{4}\right)=0,\end{cases} \\
& P(\mathbf{x})= \begin{cases}1, & \text { if } x_{3}=0, \\
x_{5}, & \text { if } x_{3} \neq 0 \& Q\left(x_{3}-3 x_{4}\right) \neq 0, \\
x_{5} x_{1}, & \text { if } x_{3} \neq 0 \& x_{3}-3 x_{4}=0, \\
-x_{5} x_{1}, & \text { if } x_{3} \neq 0 \& x_{3}-3 x_{4}=1, \\
x_{5}+1, & \text { if } x_{3} \neq 0 \& x_{3}-3 x_{4}=2 .\end{cases}
\end{aligned}
$$

Lemma 4.12. Let $K$ be a field of characteristic zero and $c \in K$. Set $\mathbf{x}_{0}=(c$, $0,0,0,1)$ and $\mathbf{x}_{i+1}=\mathbf{p}\left(\mathbf{x}_{i}\right)$ for $i \geqslant 0$. Then every entry of the sequence $\left(x_{5, i}\right)_{i \in \mathbb{N}}$ is either equal to 1 or equal to $P_{a}(c)$ for some $a=\left(a_{m}, \ldots, a_{0}\right) \in\{0,1,2\}^{m+1}$. Moreover, for $N \geqslant 1$, every $P_{N}(c)$ eventually occurs in the sequence $\left(x_{5, i}\right)_{i \in \mathbb{N}}$.

Proof. The sequence $\left(x_{1, i}\right)_{i \in \mathbb{N}}$ is constant with value $c$. The entries of the sequence $\left(x_{2, i}\right)_{i \in \mathbb{N}}$ are in $\mathbb{N}$ and in the step $i \rightsquigarrow i+1$ the sequence remains constant or increases by one. We shall see that $\left(x_{2, i}\right)_{i \in \mathbb{N}}$ eventually assumes every $N \in \mathbb{N}$. The sequences $\left(x_{3, i}\right)_{i \in \mathbb{N}}$ and $\left(x_{4, i}\right)_{i \in \mathbb{N}}$ also only take values in $\mathbb{N}$.

Note that if $x_{3, i} \neq 0$ and $Q\left(x_{3, i}-3 x_{4, i}\right) \neq 0$, then in the step $i \rightsquigarrow i+1$, the value for $x_{4}$ increases by 1 but the values of all the other $x_{i}$ 's remain constant. Let us analyze what happens in the steps $i \rightsquigarrow i+1 \rightsquigarrow i+2 \ldots$ when $x_{3, i}=0$. Then the value for $x_{2}$ increases by 1 , say $x_{2, i+1}=N \geqslant 1$. We have

$$
\mathbf{x}_{i+1}=(c, N, N, 0,1), \quad \mathbf{x}_{i+2}=(c, N, N, 1,1), \quad \mathbf{x}_{i+3}=(c, N, N, 2,1), \ldots
$$

and this continues until we reach an $\ell_{1} \geqslant 1$ such that $a_{0}=N-3 x_{4, \ell_{1}} \in\{0,1,2\}$, that is, until $x_{4, \ell_{1}}=\left\lfloor\frac{N}{3}\right\rfloor$. Note that $a_{0}=N-3 x_{4, \ell_{1}}$ is the last coefficient in the base 3 expansion $N=a_{m} 3^{m}+\cdots+a_{1} 3+a_{0}$ of $N$. So $\mathbf{x}_{\ell_{1}}=\left(c, N, N,\left\lfloor\frac{N}{3}\right\rfloor, 1\right)$ and 
because $x_{3, \ell_{1}}-3 x_{4, \ell_{1}}=a_{0} \in\{0,1,2\}$, we have $x_{3, \ell_{1}} \neq 0$ and $Q\left(x_{3, \ell_{1}}-3 x_{4, \ell_{1}}\right)=0$. Thus, according to the definition of $\mathbf{p}$ :

$$
\begin{aligned}
& \mathbf{x}_{\ell_{1}+1}=\left(c, N,\left\lfloor\frac{N}{3}\right\rfloor, 0, P_{a_{0}}(c)\right), \quad \mathbf{x}_{\ell_{1}+2}=\left(c, N,\left\lfloor\frac{N}{3}\right\rfloor, 1, P_{a_{0}}(c)\right), \\
& \mathbf{x}_{\ell_{1}+3}=\left(c, N,\left\lfloor\frac{N}{3}\right\rfloor, 2, P_{a_{0}}(c)\right), \ldots
\end{aligned}
$$

and this continues until we reach an $\ell_{2} \geqslant \ell_{1}$ such that $a_{1}=\left\lfloor\frac{N}{3}\right\rfloor-3 x_{4, \ell_{2}} \in\{0,1$, $2\}$, that is, until $x_{4, \ell_{2}}=\left\lfloor\frac{\left\lfloor\frac{N}{3}\right\rfloor}{3}\right\rfloor$. So $\mathbf{x}_{\ell_{2}}=\left(c, N,\left\lfloor\frac{N}{3}\right\rfloor,\left\lfloor\frac{\left\lfloor\frac{N}{3}\right\rfloor}{3}\right\rfloor, P_{a_{0}}(c)\right)$ and because $x_{3, \ell_{2}}-3 x_{4, \ell_{2}}=a_{1} \in\{0,1,2\}$, we have

$$
\begin{aligned}
& \mathbf{x}_{\ell_{2}+1}=\left(c, N,\left\lfloor\frac{\left\lfloor\frac{N}{3}\right\rfloor}{3}\right\rfloor, 0, P_{\left(a_{1}, a_{0}\right)}(c)\right), \\
& \mathbf{x}_{\ell_{2}+2}=\left(c, N,\left\lfloor\frac{\left\lfloor\frac{N}{3}\right\rfloor}{3}\right\rfloor, 1, P_{\left(a_{1}, a_{0}\right)}(c)\right), \ldots
\end{aligned}
$$

and so on, until we eventually reach an $\ell_{m}$ with $\ell_{m} \geqslant \ell_{m-1} \geqslant \cdots \geqslant \ell_{1}, a_{m-1}=$ $x_{3, \ell_{m}}-3 x_{4, \ell_{m}} \in\{0,1,2\}$ and $a_{m}=x_{4, \ell_{m}} \in\{1,2\}$. (The case $x_{4, \ell_{m}}=0$ does not occur because it contradicts the minimality of $\ell_{m}$.) Then

$$
\mathbf{x}_{\ell_{m}}=\left(c, N, a_{m} 3+a_{m-1}, a_{m}, P_{\left(a_{m-2}, \ldots, a_{0}\right)}(c)\right)
$$

and because $x_{3, \ell_{m}}-3 x_{4, \ell_{m}}=a_{m-1} \in\{0,1,2\}$, we have

$$
\mathbf{x}_{\ell_{m}+1}=\left(c, N, a_{m}, 0, P_{\left(a_{m-1}, \ldots, a_{0}\right)}(c)\right) \text {. }
$$

Since $x_{3, \ell_{m}+1}-3 x_{4, \ell_{m}+1}=a_{m} \in\{1,2\}$, it follows from the definition of $\mathbf{p}$ that

$$
\mathbf{x}_{\ell_{m}+2}=\left(c, N, 0,0, P_{\left(a_{m}, \ldots, a_{0}\right)}(c)\right)
$$

and

$$
\mathbf{x}_{\ell_{m}+3}=(c, N+1, N+1,0,1) .
$$

Thus the whole process repeats with $N$ incremented by 1 . Since $N=a_{m} 3^{m}+$ $\cdots+a_{0}$, the claim follows.

Lemmas 4.12 and 4.11 imply the following corollary.

COROLlARY 4.13. With notation as in Lemma 4.12, we have the following: The sequence $\left(x_{5, i}\right)_{i \in \mathbb{N}}$ contains zero if and only if c is algebraic over $\mathbb{Q}$. 
We are now prepared to prove Theorem 3.2.

Proof of Theorem 3.2. As above, we consider the piecewise polynomial map p: $\mathbb{A}_{\mathbb{Q}}^{5} \rightarrow \mathbb{A}_{\mathbb{Q}}^{5}$ given by $\mathbf{p}=(C, N, R, A, P)$ with $C, N, R, A, P$ defined in equation (8). Let $V$ denote the closed subset of $\mathbb{A}_{\mathbb{Q}}^{5}$ defined by $X_{2}=X_{3}=X_{4}=$ $0, X_{5}=1$. According to Lemma 4.6, there exists an integer $r \geqslant 1$, a finite system $F=\left\{f_{1}, \ldots, f_{\ell}\right\} \subseteq \mathbb{Q}\left[\sigma^{i}\left(T_{1}\right), \ldots, \sigma^{i}\left(T_{r}\right) \mid i \in \mathbb{N}\right]$, and a difference polynomial $g \in \mathbb{Q}\left[\sigma^{i}\left(T_{1}\right), \ldots, \sigma^{i}\left(T_{r}\right) \mid i \in \mathbb{N}\right]$ such that, for every field extension $K$ of $\mathbb{Q}$, the following two statements are equivalent:

(i) There exists a sequence $\left(\mathbf{x}_{i}\right)_{i \in \mathbb{N}}=\left(x_{1, i}, \ldots, x_{5, i}\right)_{i \in \mathbb{N}} \in\left(K^{\mathbb{N}}\right)^{5}$ such that

$$
\mathbf{x}_{0} \in V(K), \quad \mathbf{x}_{i+1}=\mathbf{p}\left(\mathbf{x}_{i}\right) \quad \text { for every } i \in \mathbb{N},
$$

and $x_{5, i} \neq 0$ for $i \geqslant 1$.

(ii) There exists a solution of $F=0$ in $\left(K^{\mathbb{N}}\right)^{r}$ such that $g$ does not vanish on this solution.

Following Corollary 4.13, we see that (i) does not hold for the field $K=\overline{\mathbb{Q}}$, whereas (i) does hold for the field $K=\mathbb{C}$ (or any transcendental extension of $\mathbb{Q})$. Thus, (for $K=\overline{\mathbb{Q}}$ ) we see that $g$ vanishes on every solution of $F=0$ in $\left(\overline{\mathbb{Q}}^{\mathbb{N}}\right)^{r}$, that is, $g \in \mathcal{I}(\mathcal{V}(F)$ ). However (for $K=\mathbb{C}$ ), it follows that $g$ does not vanish on every solution of $F=0$ in $\left(\mathbb{C}^{\mathbb{N}}\right)^{r}$. Since an element of $\sqrt{\left\langle\sigma^{i}(F) \mid i \in \mathbb{N}\right\rangle}$ vanishes on every solution of $F=0$ over any field extension of $\mathbb{Q}$, we deduce that $g \notin \sqrt{\left\langle\sigma^{i}(F) \mid i \in \mathbb{N}\right\rangle}$.

\section{Acknowledgements}

The authors would like to thank Olivier Bournez, Ivan Mitrofanov, Alexey Ovchinnikov, and Amaury Pouly for helpful discussions. The authors thank the anonymous referee for a close reading of an earlier version of this paper and for suggesting some improvements. This work has been partially supported by NSF grants CCF-1564132, CCF-1563942, DMS-1760448, DMS-1760212, DMS1760413, DMS-1853482, DMS-1853650; by PSC-CUNY grants \#69827-0047, \#60098-0048; and by the Lise Meitner grant M 2582-N32 of the Austrian Science Fund FWF.

Conflict of Interest: None. 


\section{References}

[1] R. Berger, 'The undecidability of the domino problem', Mem. Amer. Math. Soc. 66 (1966), $1-72$.

[2] O. Bournez and A. Pouly, Handbook of Computability and Complexity in Analysis, A Survey on Analog Models of Computation (Springer, 2018), to appear, https://arxiv.org/abs/1805.05 729.

[3] R. F. Bustamante Medina, 'Differentially closed fields of characteristic zero with a generic automorphism', Rev. Mat. Teor. Apl. 14(1) (2007), 81-100.

[4] Z. Chatzidakis, 'Model theory of fields with operators - a survey', in Logic Without Borders - Essays on Set Theory, Model Theory, Philosophical Logic and Philosophy of Mathematics (Walter de Gruyter, Berlin/Boston/Munich, 2015), 91-114.

[5] Z. Chatzidakis and E. Hrushovski, 'Model theory of difference fields', Trans. Amer. Math. Soc. 351(8) (1999), 2997-3071.

[6] R. Cohn, Difference Algebra (Interscience Publishers John Wiley \& Sons, New YorkLondon-Sydney, 1965).

[7] G. Everest, A. van der Poorten, I. Shparlinski and T. Ward, Recurrence Sequences (American Mathematical Society, Providence, RI, 2003).

[8] X.-S. Gao, Y. Luo and C. Yuan, 'A characteristic set method for ordinary difference polynomial systems', J. Symbolic Comput. 44(3) (2009), 242-260.

[9] X. S. Gao, J. van der Hoeven, C. M. Yuan and G. L. Zhang, 'Characteristic set method for differential-difference polynomial systems', J. Symbolic Comput. 44(9) (2009), 1137-1163.

[10] G. H. Hardy and E. M. Wright, An Introduction to the Theory of Numbers, 6th edn, (Oxford University Press, 2008).

[11] H. A. Heilbronn, 'On discrete harmonic functions', Math. Proc. Cambridge Philos. Soc. 45(2) (1949), 194-206.

[12] E. Hrushovski, 'The Manin-Mumford conjecture and the model theory of difference fields', Ann. Pure Appl. Logic 112(1) (2001), 43-115.

[13] E. Hrushovski and F. Point, 'On von Neumann regular rings with an automorphism', J. Algebra 315(1) (2007), 76-120.

[14] Z. Jelonek, 'On the effective Nullstellensatz', Invent. Math. 162(1) (2005), 1-17.

[15] H. Kikyo, 'On generic predicates and automorphisms', RIMS K^oky uroku Bessatsu 1390 (2004), 1-8.

[16] P. Koiran and C. Moore, 'Closed-form analytic maps in one and two dimensions can simulate universal Turing machines', Theor. Comput. Sci. 210(1) (1999), 217-223.

[17] E. R. Kolchin, Differential Algebra and Algebraic Groups (Academic Press, New York, 1973).

[18] S. Lang, 'Hilbert's Nullstellensatz in infinite-dimensional space', Proc. Amer. Math. Soc. 3 (1952), 407-410.

[19] O. Léon Sánchez, 'On the model companion of partial differential fields with an automorphism', Israel J. Math. 212(1) (2016), 419-442.

[20] A. Levin, Difference Algebra (Springer, The Netherlands, 2008).

[21] G. S. Makanin, 'The problem of solvability of equations in a free semigroup', Math. USSR Sbornik 32(2) (1977), 129-198.

[22] Y. V. Matijasevic, 'Enumerable sets are Diophantine', Soviet Math. Dokl. 11 (1970), 354-357.

[23] C. Moore, 'Unpredictability and undecidability in dynamical systems', Phys. Rev. Lett. 64 (1990), 2354-2357. 
[24] A. Ovchinnikov, G. Pogudin and T. Scanlon, Effective difference elimination and Nullstellensatz. Accepted for publication in the J. Eur. Math. Soc., (2019).

[25] J. Ritt, Differential Algebra (American Mathematical Society, Providence, RI, 1950).

[26] C. P. Schnorr, 'A unified approach to the definition of random sequences', Math. Sys. Theory 5(3) (1971), 246-258.

[27] M. F. Singer, 'The model theory of ordered differential fields', J. Symbolic Logic 43(1) (1978), 82-91.

[28] A. Tarski, A Decision Method for Elementary Algebra and Geometry, (RAND Corporation, Santa Monica, CA, 1948).

[29] U. Umirbaev, 'Algorithmic problems for differential polynomial algebras', J. Algebra 455 (2016), 77-92. 